archives-ouvertes

\title{
Influence of condensation and latent heat release upon barotropic and baroclinic instabilities of vortices in a rotating shallow water f-plane model
}

Masoud Rostami, Vladimir Zeitlin

\section{To cite this version:}

Masoud Rostami, Vladimir Zeitlin. Influence of condensation and latent heat release upon barotropic and baroclinic instabilities of vortices in a rotating shallow water f-plane model. Geophysical and Astrophysical Fluid Dynamics, Taylor \& Francis, 2017, 111 (1), pp.1-31. $<10.1080 / 03091929.2016 .1269897>$. <hal-01433707>

HAL Id: hal-01433707

\section{http://hal.upmc.fr/hal-01433707}

Submitted on 15 Jun 2017

HAL is a multi-disciplinary open access archive for the deposit and dissemination of scientific research documents, whether they are published or not. The documents may come from teaching and research institutions in France or abroad, or from public or private research centers.
L'archive ouverte pluridisciplinaire HAL, est destinée au dépôt et à la diffusion de documents scientifiques de niveau recherche, publiés ou non, émanant des établissements d'enseignement et de recherche français ou étrangers, des laboratoires publics ou privés. 
This is the Authors' Original Manuscript (AOM); that is, the manuscript in its original form; a 'preprint'. The Version of Record (VOR) of this manuscript has been published and is available in the: "Geophysical \& Astrophysical Fluid Dynamics".

Published online: 4 Jan 2017

http://dx.doi.org/10.1080/03091929.2016.1269897

DOI: $10.1080 / 09500693.2015 .1022623$ 
This is the Authors' Original Manuscript (AOM); that is, the manuscript in its original form; a ' preprint. The Version of Record (VOR) of this manuscript has been published and is available in the: "Geophysical \& Astrophysical Fluid Dynamics" .

Published online: 4 Jan 2017

http://dx.doi.org/10.1080/03091929.2016.1269897

DOI: $10.1080 / 09500693.2015 .1022623$

\title{
Influence of condensation and latent heat release upon barotropic and baroclinic instabilities of vortices in rotating shallow water $f$-plane model
}

\author{
MASOUD ROSTAMI ${ }^{\dagger}$ and VLADIMIR ZEITLIN ${ }^{\dagger}$ \\ † Laboratoire de Météorologie Dynamique/Université Pierre et Marie Curie (UPMC)/ Ecole Normale \\ Supérieure (ENS)/CNRS, Paris, France \\ (Received 00 Month 20xx; final version received 00 Month 20xx)
}

\begin{abstract}
Analysis of the influence of condensation and related latent heat release upon developing barotropic and baroclinic instabilities of large-scale low Rossby-number shielded vortices on the $f$ - plane is performed within the moist-convective rotating shallow water model, in its barotropic (one-layer) and baroclinic (two-layer) versions. Numerical simulations with a high-resolution well-balanced finite-volume code, using a relaxation parameterisation for condensation, are made. Evolution of the instability in four different environments, with humidity (i) behaving as passive scalar, (ii) subject to condensation beyond a saturation threshold, (iii) subject to condensation and evaporation, with two different parameterisations of the latter, are inter-compared. The simulations are initialised with unstable modes determined from the detailed linear stability analysis in the "dry" version of the model. In a configuration corresponding to low-level mid-latitude atmospheric vortices, it is shown that the known scenario of evolution of barotropically unstable vortices, consisting in formation of a pair of dipoles ("dipolar breakdown") is substantially modified by condensation and related moist convection, especially in the presence of surface evaporation. No enhancement of the instability due to precipitation was detected in this case. Cyclone-anticyclone asymmetry with respect to sensitivity to the moist effects is evidenced. It is shown that inertia-gravity wave emission during the vortex evolution is enhanced by the moist effects. In the baroclinic configuration corresponding to idealised cut-off lows in the atmosphere, it is shown that the azimuthal structure of the leading unstable mode is sensitive to the details of stratification. Scenarios of evolution are completely different for different azimuthal structures, one leading to dipolar breaking, and another to tripole formation. The effects of moisture considerably enhance the perturbations in the lower layer, especially in the tripole formation scenario.
\end{abstract}

Keywords: Moist-convective Rotating Shallow Water, Vortex Dynamics, Barotropic Instability, Baroclinic Instability

\section{Introduction}

The purpose of the present paper is to understand how the effects of moist convection and condensation affect instabilities and evolution of large-scale atmospheric vortices. Our main interest is in the impact of condensation and related latent heat release upon dynamics, so we will not have to recourse to the full-scale thermodynamics of the moist air and will be using a simplified model where only the most rough features of the moist convection are taken into account. Dynamically, large-scale low Rossby-number vortices, which we will be considering in the $f$-plane approximation, are well-described within the quasi-geostrophic (QG) models, where the effects of moist convection may be included in a simple way, on the basis of conservation of the moist potential vorticity (Lapeyre and Held 2004). Yet, by construction, QG models miss an important dynamical ingredient, inertia-gravity waves (IGW). (Another element which the QG model misses is sharp density/potential temperature fronts, although those are out of the scope of the present paper). Vortex instabilities are known to produce IGW emission, and its quantification is important in the general context of understanding the sources of IGW in the atmosphere. That is why we choose to work with the so-called moistconvective rotating shallow water model $(\mathrm{mcRSW})$ which incorporates the moist convection 
and condensation in a simple, albeit self-consistent, way. As usual, the QG equations may be recovered in the model in the limit of small Rossby numbers. The model in its barotropic and baroclinic versions, respectively, was proposed in (Bouchut et al. 2009, Lambaerts et al. 2011a) and was inspired by the pioneering work by Gill (1982). The work of Ooyama (1969) was probably the first where such kind of model, in axisymmetric version, was applied for studying dynamics of atmospheric vortices - tropical cyclones. This approach was later pursued by Zehnder (2001). A similar model, with special attention to the parameterisation of the boundary layer processes, was used by Schecter (2009) for understanding tropical cyclogeneses. Recently, the barotropic version of the model was applied to modelling the development of instabilities of tropical cyclones (Lahaye and Zeitlin 2016).

Unlike the latter papers dealing with intense vortices with high Rossby numbers, we will be using the model for studying instabilities of large-scale small Rossby number barotropic and baroclinic vortices and their nonlinear dynamical saturation (it should be emphasized that the term "saturation" is used below to describe both the saturation of the moist air in the thermodynamical sense, and also dynamical saturation of the instability in the sense that growth predicted by linear analysis ceases and gives rise to reorganisation of the flow). To quantify dynamical influence of moisture, we will be comparing the behavior of vortices in "dry" and "moist-precipitating" configurations of the model, with the moisture being a passive tracer in the former (which is thus, in fact, moist (M), but not precipitating) and having a condensation sink (MP) which creates a moist-convective vertical flux in the latter. (We should recall that, in the framework of mcRSW, condensation and precipitation are synonymous.) Adding evaporation source gives a third, moist-precipitating-evaporating (MPE) configuration, which will be also studied. Our strategy will be the same as that of (Lambaerts et al. 2011b, 2012), in studying dynamical influence of moisture upon instabilities of barotropic and baroclinic jets. A notable difference with these papers, though, is that in the present study we also include the effects of surface evaporation, which will be shown to be important. However, we will be not dwelling into details of the boundary-layer processes, and will be limiting ourselves by the simplest possible parameterisations of fluxes across the lower boundary of the model.

It should be stressed that condensation and related moist convection are essentially nonlinear phenomena, and hence the techniques of linear stability analysis are inapplicable in the moist-precipitating case. So, as in the case of jets (Lambaerts et al. 2011b, 2012), we will be performing linear stability analysis of "dry" vortices, and then using the obtained unstable modes to initialise numerical simulations of both "dry" and moist-precipitating (and evaporating) evolution of the instability. The well-balanced high-resolution finite-volume numerical scheme adapted for mcRSW in (Bouchut et al. 2009, Lambaerts et al. 2011a), will be used in these simulations. The model resolves well the IGW, including front (shock) formation, the precipitation fronts, and maintains balanced states. It also allows for self-consistent inclusion of topography, which is however out of the scope of the present paper.

By construction the one-layer version of the model is a limit of the two-layer one with infinitely deep upper layer, see below. So, physically, vortices in the one-layer model represent idealised low-level atmospheric vortices, while the baroclinic vortices that we treat in the twolayer model are upper-level vortices, of the type of cut-off lows frequently encountered in the atmosphere at mid-latitudes.

The paper is organised as follows. The model, both in one- and two-layer versions, and the vortex configurations to be studied are introduced in section 2 . Linear stability analysis framework and results are presented in section 3. Section 4 contains results on nonlinear evolution of the barotropic and baroclinic vortex instabilities in "dry", moist-precipitating and moist-precipitating and evaporating cases, and their inter-comparison. Conclusions and discussion are presented in section 5. 


\section{The model and the background flow}

In this section we present the model we are working with. It is a rotating shallow water model where convective fluxes due to the latent heat release are added at the interface between the lower, humid, and the upper, dry layer, as well as surface evaporation at the lower boundary. Although multi-layer generalisation of the model is possible, cf. the references in sect. 1 above, we will be using the two- layer version, and its one-layer reduction. The model retains the most rough features of the moist convection, yet in a self-consistent way, and allows to follow both vortex and inertia-gravity wave components of the flow.

\subsection{Moist-convective rotating shallow water model}

\subsubsection{Equations of motion}

The equations of the two-layer mcRSW, as introduced in (Lambaerts et al.|2011a) are:

$$
\left\{\begin{array}{l}
\frac{D_{1} \boldsymbol{v}_{1}}{D t}+f \hat{z} \times \boldsymbol{v}_{1}=-g \nabla_{H}\left(h_{1}+h_{2}\right), \\
\frac{D_{2} \boldsymbol{v}_{2}}{D t}+f \hat{z} \times \boldsymbol{v}_{2}=-g \nabla_{H}\left(h_{1}+s h_{2}\right)+\frac{\boldsymbol{v}_{1}-\boldsymbol{v}_{2}}{h_{2}} \beta P_{1}, \\
\partial_{t} h_{1}+\nabla \cdot\left(h_{1} \boldsymbol{v}_{1}\right)=-\beta P_{1}, \\
\partial_{t} h_{2}+\nabla \cdot\left(h_{2} \boldsymbol{v}_{2}\right)=+\beta P_{1}, \\
\partial_{t} Q_{1}+\nabla \cdot\left(Q_{1} \boldsymbol{v}_{1}\right)=-P_{1}+E .
\end{array}\right.
$$

Here $\boldsymbol{v}_{i}$ is the horizontal velocity field in layer $i=1,2$ (counted from the bottom) and $D_{i} / D t$ is the corresponding horizontal material derivative. $f$ is the Coriolis parameter, which will be supposed to be constant, $\hat{z}$ is the unit vector in $z$-direction, $h_{i}$ are thicknesses of the layers, $\theta_{i}$ is the normalised potential temperature in each layer, $s=\left(\theta_{2} / \theta_{1}\right)>1$ is the stratification parameter. Let us recall that this model is obtained from the primitive equations, with pseudo-height as vertical coordinate, by vertical averaging between the material surfaces $z_{i-1}, z_{i}$, with $z_{i}-z_{i-1}=h_{i}$ and adding additional convective flux due to the latent heat release through $z_{1}$. This convective flux is then linked to the water-vapour condensation $P_{1}$ in the lower humid layer through the moist enthalpy conservation, and gives a sink (source) in the mass conservation equation in the lower (upper) layer. The coefficient $\beta$ is determined by the background stratification in the parent primitive-equations model, see Lambaerts et al. (2011a). Due to the convective mass exchange the same flux leads to appearance of the Rayleigh drag in the upper-layer momentum equation. At the same time, condensation gives a sink in the bulk moisture content $Q_{1}$ in the lower layer. If surface evaporation $E$ is present, it provides a source of moisture in the lower layer. Finally, condensation and moisture content are related by a simple relaxation relation:

$$
P_{1}=\frac{Q_{1}-Q^{s}}{\tau} H\left(Q_{1}-Q^{s}\right),
$$

where $H($.$) is the Heaviside (step) function, and Q^{s}$ is a saturation value. This scheme is of the type used in general circulation models (Betts and Miller 1986). In what follows the simplest parameterisation with a constant $Q^{s}$ is used. Note that the condensed water is dropped off the dynamics, and we do not consider the inverse phase transition liquid water - water vapor in this simplified model. (Hence the evaporation $E$, if any, is the surface one in the model, and in this sense there is no difference between condensation and precipitation, and they will 
be used as synonyms in what follows). Radiation cooling will be omitted as well, cf. Ooyama (1969).

It is useful to consider the limit $\tau \rightarrow 0$ in (2), because the relaxation time $\tau$ is small (several hours) in the atmosphere, and will be small (several time-steps) in the numerical simulations presented below. As shown in (Gill 1982, Bouchut et al. 2009), in this (wet) limit

$$
P=-Q^{s} \nabla \cdot v,
$$

and precipitation is directly proportional to the wind convergence. There exist several simple parameterisations for surface evaporation, for example, proportional to the deviation of the local value of humidity from the saturation: $E=E 1=\gamma\left(Q_{s}-Q\right) H\left(Q_{s}-Q\right)$, or proportional to the wind velocity: $E=E 2=\delta|\boldsymbol{v}|$, where coefficients $\gamma$ and $\delta$ are adjustable parameters. Both were used in the literature, e.g. (Neelin et al.|1987, Goswami and Goswami|1991), the first more adapted for situations with weak winds, and the second - to strongly under-saturated boundary layer. The two may be combined, cf. e.g. (Ooyama 1969), (Kondo et al. 1990): $E=E 3=\kappa|\boldsymbol{v}|\left(Q_{s}-Q\right) H\left(Q_{s}-Q\right)$. We will be testing all three of them below.

In the limit of very deep upper layer $h_{2} \rightarrow \infty$ we obtain a simplified one-layer version of the model :

$$
\left\{\begin{array}{l}
\partial_{t} \boldsymbol{v}_{1}+\left(\boldsymbol{v}_{1} \cdot \nabla\right) \boldsymbol{v}_{1}+f \hat{z} \times \boldsymbol{v}_{1}=-g \nabla h_{1}, \\
\partial_{t} h_{1}+\nabla \cdot\left(\boldsymbol{v}_{1} h_{1}\right)=-\beta P_{1}, \\
\partial_{t} Q_{1}+\nabla \cdot\left(Q_{1} \boldsymbol{v}_{1}\right)=-P_{1}+E,
\end{array}\right.
$$

see Lambaerts et al. (2011a) for a detailed demonstration.

\subsubsection{Conservation laws}

Conservation laws of the standard RSW model change in the presence of precipitation and related convection. Although mass and bulk humidity are not conserved in the lower layer, their combination $m_{1}=h_{1}-\beta Q$, corresponding in this simplified model to the moist enthalpy, is locally conserved in the absence of surface evaporation:

$$
\partial_{t} m_{1}+\nabla \cdot\left(m_{1} \boldsymbol{v}_{1}\right)=0 .
$$

This shows the consistency of the model, in spite of its simplicity. Potential vorticity (PV) equations in each layer in the presence of precipitation and without evaporation, $E=0$, become:

$$
\begin{aligned}
& \left(\partial_{t}+\boldsymbol{v}_{1} \cdot \nabla\right) \frac{\zeta_{1}+f}{h_{1}}=\frac{\zeta_{1}+f}{h_{1}^{2}} \beta P_{1}, \\
& \left(\partial_{t}+\boldsymbol{v}_{2} \cdot \nabla\right) \frac{\zeta_{2}+f}{h_{2}}=-\frac{\zeta_{2}+f}{h_{2}^{2}} \beta P_{1}+\frac{\hat{z}}{h_{2}} \cdot\left\{\nabla \times\left(\frac{\boldsymbol{v}_{1}-\boldsymbol{v}_{2}}{h_{2}} \beta P_{1}\right)\right\},
\end{aligned}
$$

where $\zeta_{i}=\hat{z} \cdot\left(\nabla \times \boldsymbol{v}_{i}\right)=\partial_{x} v_{i}-\partial_{y} u_{i}(i=1,2)$ is relative vorticity, and $q_{i}=\left(\zeta_{i}+f\right) / h_{i}, i=1,2$ is $\mathrm{PV}$ layerwise. Hence, PV in each layer is not a Lagrangian invariant in precipitating regions. In the absence of evaporation, the conservation of the moist enthalpy in the lower layer allows to derive a new Lagrangian invariant, the moist PV:

$$
\left(\partial_{t}+\boldsymbol{v}_{1} \cdot \nabla\right) \frac{\zeta_{1}+f}{m_{1}}=0 .
$$

It should be noted that surface evaporation renders the system forced, and destroys the conservation of moist enthalpy and moist potential vorticity. Care should be taken in numerical simulations with evaporation, as moist enthalpy should remain everywhere positive to ensure thermodynamic stability. The dry energy of the system is $\mathcal{E}=\int d x d y\left(\mathrm{e}_{1}+\mathrm{e}_{2}\right)$, where the 
energy densities of the layers are:

$$
\left\{\begin{array}{l}
\mathrm{e}_{1}=h_{1} \frac{\boldsymbol{v}_{1}^{2}}{2}+g \frac{h_{1}^{2}}{2} \\
\mathrm{e}_{2}=h_{2} \frac{\boldsymbol{v}_{2}^{2}}{2}+g h_{1} h_{2}+s g \frac{h_{2}^{2}}{2} .
\end{array}\right.
$$

Supposing that there are no energy exchanges through the boundaries, we get:

$$
\partial_{t} \mathcal{E}=-\int d x d y \beta P\left(g h_{2}(1-s)+\frac{\left(\boldsymbol{v}_{1}-\boldsymbol{v}_{2}\right)^{2}}{2}\right) .
$$

The first term in this equation corresponds to production of potential energy (for stable stratifications) due to upward convection fluxes; the second term corresponds to destruction of kinetic energy due to the Rayleigh drag.

The horizontal momentum of individual layers is not conserved due to convective mass exchanges, but the total momentum of the two-layer system is conserved, cf (Lambaerts et al. 2011a).

\section{2. $\quad$ Vortex configurations}

We start with a simpler case of one-layer model, which will also serve for benchmarking. Anticipating the use of axisymmetric solutions, we write down the "dry" $\left(P_{1}=0\right)$ version of (4) in polar coordinates $(r, \theta)$ in terms of radial and azimuthal components of the velocity $\boldsymbol{v}=u \hat{r}+v \hat{\theta}$, where hats denote unit vectors in corresponding directions:

$$
\left\{\begin{array}{l}
\frac{D u}{D t}-\frac{v^{2}}{r}-f v=-g \partial_{r} h, \\
\frac{D v}{D t}+\frac{u v}{r}+f u=-g \partial_{\theta} h, \\
\partial_{t} h+\frac{1}{r} \partial_{r}(h r u)+\frac{1}{r} \partial_{\theta}(h v)=0 .
\end{array}\right.
$$

Here $D / D t$ is the horizontal material derivative in polar coordinates (we omit the subscript 1 from now on). As is clear from these equations, and well-known, any axisymmetric flow (vortex) with azimuthal velocity $v=V(r)$ and thickness $h=H(r)$ in cyclo-geostrophic equilibrium (gradient wind balance in the language of meteorology)

$$
\left(\frac{V}{r}+f\right) V=g \partial_{r} H
$$

is an exact solution of 10 . In what follows we are interested in isolated vortices, i.e. those satisfying (11) and having zero circulation at infinity. It is simpler to work with non-dimensional variables and we will be using the following scaling: $\sqrt{g H_{0}}$ for velocity, $R_{d}=\sqrt{g H_{0}} / f$ for $r$, and $1 / f$ for time, where $H_{0}$ is the non-perturbed thickness of the layer. Star notation will be adopted for non-dimensional variables. We choose to work with so-called $\alpha$-Gaussian vortices with the following non-dimensional distribution of azimuthal velocity (non-dimensional variables are denoted by an asterisk):

$$
V^{*}\left(r^{*}\right)= \pm \epsilon r^{* \frac{\alpha}{2}} \exp \left(\frac{-r^{* \alpha}+1}{2}\right), \quad \alpha \geq 1 .
$$

Here the positive sign corresponds to cyclones and the negative one to anticyclones. The corresponding profile of $H\left(r^{*}\right)$ is given by the primitive of the l.h.s. of (11) calculated with 
12):

$$
H^{*}\left(r^{*}\right)=1-( \pm) \epsilon \frac{1}{\alpha} \sqrt{2 \mathrm{e}} 2^{\frac{1}{\alpha}} \Gamma\left(\frac{1}{\alpha}+\frac{1}{2}\right) G\left(\frac{r^{* \alpha}}{2}, \frac{1}{\alpha}+\frac{1}{2}\right), \text { where } \quad G(x, a)=\frac{1}{\Gamma(a)} \int_{x}^{a} \mathrm{e}^{-t} t^{a-1} d t .
$$

The $\alpha$-Gaussian vortices have two parameters: $\alpha$ and $\epsilon$, which control the steepness of the azimuthal velocity profile and the amplitude of the velocity, respectively. This class of vortices was used in recent studies of vortex instabilities (Kloosterziel et al. 2007, Lahaye and Zeitlin 2015). It is slightly different from another profile, also used in the literature (Carton et al. 1989, Baey and Carton 2002), but the two coincide at $\alpha=2$. Such profiles provide a simple analytical form of a shielded (i.e. with zero circulation at infinity, and hence finite energy) vortex. Radial distribution of the relative vorticity in the vortex is given by $\left(1 / r^{*}\right) d\left(r^{*}\left(V^{*}\left(r^{*}\right)\right)\right) / d r^{*}$, therefore cyclonic vortices have a core of positive relative vorticity inside a ring of negative relative vorticity, and vice verse for anticyclonic vortices. So we deal with vortices that possess a sign reversal in the radial vorticity profile, which should produce a barotropic instability, according to well-known criteria. We focus below on vortices with small Rossby numbers, i.e. with peak azimuthal velocities of small amplitude. If we recall the interpretation of the one-layer model as a two-layer model with infinitely deep upper layer, the above configuration corresponds to a vortex in the lower layer with motionless upper layer. An opposite situation of a vortex with the same profile in the upper layer and motionless lower layer can be considered in the two-layer version of the model. Such upper-layer vortices, so called cut-off lows, e.g. (Gimeno et al. 2007), are frequently produced in the atmosphere in mid-latitudes by meandering uppertropospheric jets. Writing the "dry" two-layer system in polar coordinates is straightforward, and the two-layer analog of $(11)$ is

$$
\left\{\begin{array}{l}
\left(\frac{V_{1}}{r}+f\right) V_{1}=g \partial_{r}\left(H_{1}+H_{2}\right), \\
\left(\frac{V_{2}}{r}+f\right) V_{2}=g \partial_{r}\left(H_{1}+s H_{2}\right) .
\end{array}\right.
$$

It is clear that taking the profile $(12)$ for $V_{2}$, supposing $H_{1}+s H_{2}=$ const, and taking $H_{1}+s H_{2}$ in the form of (13), allows to find unambiguously $H_{1}$ and $H_{2}$ for an upper-layer vortex.

\section{Linear stability analysis}

In this section we formulate the linear stability problem, sketch the method of its solution, and present results of the linear stability analysis of the alpha -Gaussian vortices in one- and two-layer versions of the model.

\subsection{Linear stability of vortices in the one-layer model}

\subsubsection{Formulation of the linear stability problem}

To analyse the linear stability of vortex solutions we apply the standard linearisation procedure by considering a small perturbation of the axisymmetric background flow: The linearised non-dimensional equations read:

$$
\left\{\begin{array}{l}
\left(\partial_{t^{*}}+\frac{V^{*}}{r^{*}} \partial_{\theta^{*}}\right) u^{*}-\left(1+\frac{2 V^{*}}{r^{*}}\right) v^{*}=-\partial_{r^{*}} \eta^{*} \\
\left(\partial_{r^{*}} V^{*}+1+\frac{V^{*}}{r^{*}}\right) u^{*}+\left(\partial_{t^{*}}+\frac{V^{*}}{r^{*}} \partial_{\theta^{*}}\right) v^{*}=-\frac{1}{r^{*}} \partial_{\theta^{*}} \eta^{*} \\
\left(\partial_{t^{*}}+\frac{V^{*}}{r^{*}} \partial_{\theta^{*}}\right) \eta^{*}+\left[H^{*} \partial_{r^{*}}+\left(\frac{1}{r^{*}} \partial_{r^{*}} r^{*} H^{*}\right)\right] u^{*}+\frac{1}{r^{*}} H^{*} \partial_{\theta^{*}} v^{*}=0
\end{array}\right.
$$


where we denote the perturbations of $u$ and $v$ by the same letters, and the perturbation of $h$ by $\eta$. We are looking for the normal-mode solutions with harmonic dependence on time and polar angle

$$
\left(u^{*}, v^{*}, \eta^{*}\right)\left(r^{*}, \theta^{*}, t^{*}\right)=\operatorname{Re}\left[(\mathrm{i} \tilde{u}, \tilde{v}, \tilde{\eta})\left(r^{*}\right) \mathrm{e}^{\mathrm{i}\left(l \theta^{*}-\omega t^{*}\right)}\right],
$$

where $l$ and $\omega$ are the azimuthal wavenumber and the frequency, respectively. Substitution of (16) in (15) yields the eigen-problem:

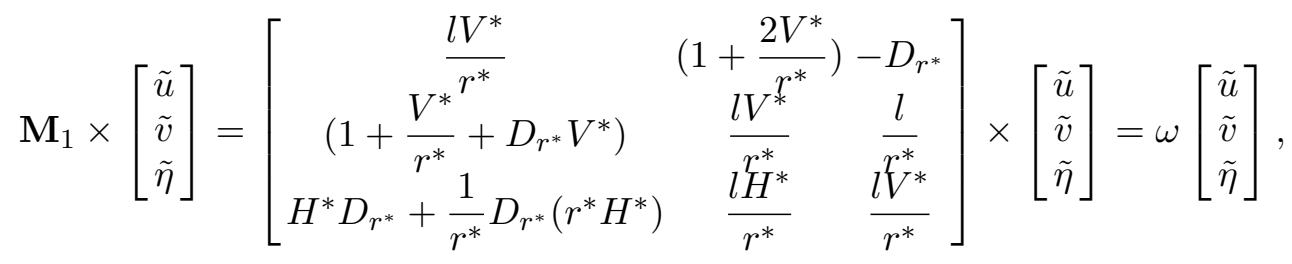

where $D_{r^{*}}$ denotes the differentiation operator with respect to $r^{*}$. Complex eigenfrequencies $\omega=\omega_{r}+\mathrm{i} \omega_{I}$ with positive imaginary part $\left(\omega_{I}>0\right)$, correspond to instabilities with linear growth rate $\sigma=\omega_{I}$. Below we perform a numerical linear stability analysis of the problem (17) with the help of the pseudo-spectral collocation method (Trefethen 2000). The system is discretised over an $N$-point grid and $D_{r^{*}}$ becomes the Chebyshev differentiation operator. To avoid the Runge phenomenon, Chebyshev collocation points are used, with a stretching in the radial direction allowing to densify the collocation points in the most dynamically interesting region near the center of the vortex, as in (Lahaye and Zeitlin 2015) where the method of Boyd (1987) was adapted to the stability problem of circular vortices. In order to make the standard parameters, Rossby and Burger numbers, to appear, an alternative scaling can be used, based on the typical horizontal scale of the background flow $L\left(r=L r^{*}\right)$. In this case, the matrix in l.h.s. of (17) becomes:

$$
\boldsymbol{M}_{1}=\left[\begin{array}{ccc}
R o \frac{l V^{*}}{r^{*}} & \left(1+R o \frac{2 V^{*}}{r^{*}}\right) & -\frac{B u}{R o} D_{r^{*}} \\
\left(1+R o \frac{V^{*}}{r^{*}}+R o . D_{r^{*}} V^{*}\right) & R o \frac{l V^{*}}{r^{*}} & \frac{1}{R o} \frac{l}{r^{*}} \\
R o\left[H^{*} D_{r^{*}}+\frac{1}{r^{*}} D_{r^{*}}\left(r^{*} H^{*}\right)\right] & R o \frac{l H^{*}}{r^{*}} & R o \frac{l V^{*}}{r^{*}}
\end{array}\right],
$$

where $R o=U / f L, B u=g H / f^{2} L^{2}=\left(R_{d} / L\right)^{2}$.

\subsubsection{Results of the linear stability analysis}

We describe in this subsection the results of the linear stability analysis of cyclonic and anticyclonic $\alpha$-Gaussian vortices obtained by pseudo-spectral collocation method, which was briefly sketched above. We present in Fig. 1 a typical output of the numerical linear stability analysis. The structure of unstable modes together with the background vortex profile of a cyclone are displayed. Results for an anti-cyclone with the same parameters are similar (not shown).

We have analysed the linear stability of cyclonic and anticyclonic vortices for the values of steepness parameter $\alpha$ varying from 2 to 6 , and the amplitude parameter $\epsilon$ varying from 0.05 to 1 , with a general result that the growth rate of the unstable modes monotonically increases with $\alpha$ and $\epsilon$, as follows from Fig. 2, right panel. The azimuthal wavenumber of the most unstable mode increases with $\alpha$, cf. Fig. 2, left and middle panels. There is a switch in the azimuthal structure of the most unstable mode when steepness increases. These results qualitatively agree with those of Baey and Carton (2002) which were obtained with a similar, but not the same, vortex profile. It is worth noting that our results show that for the same values of parameters, the growth rates of the most unstable modes are higher for cyclonic than for anticyclonic vortices, which shows cyclone-anticyclone asymmetry already at this level. 

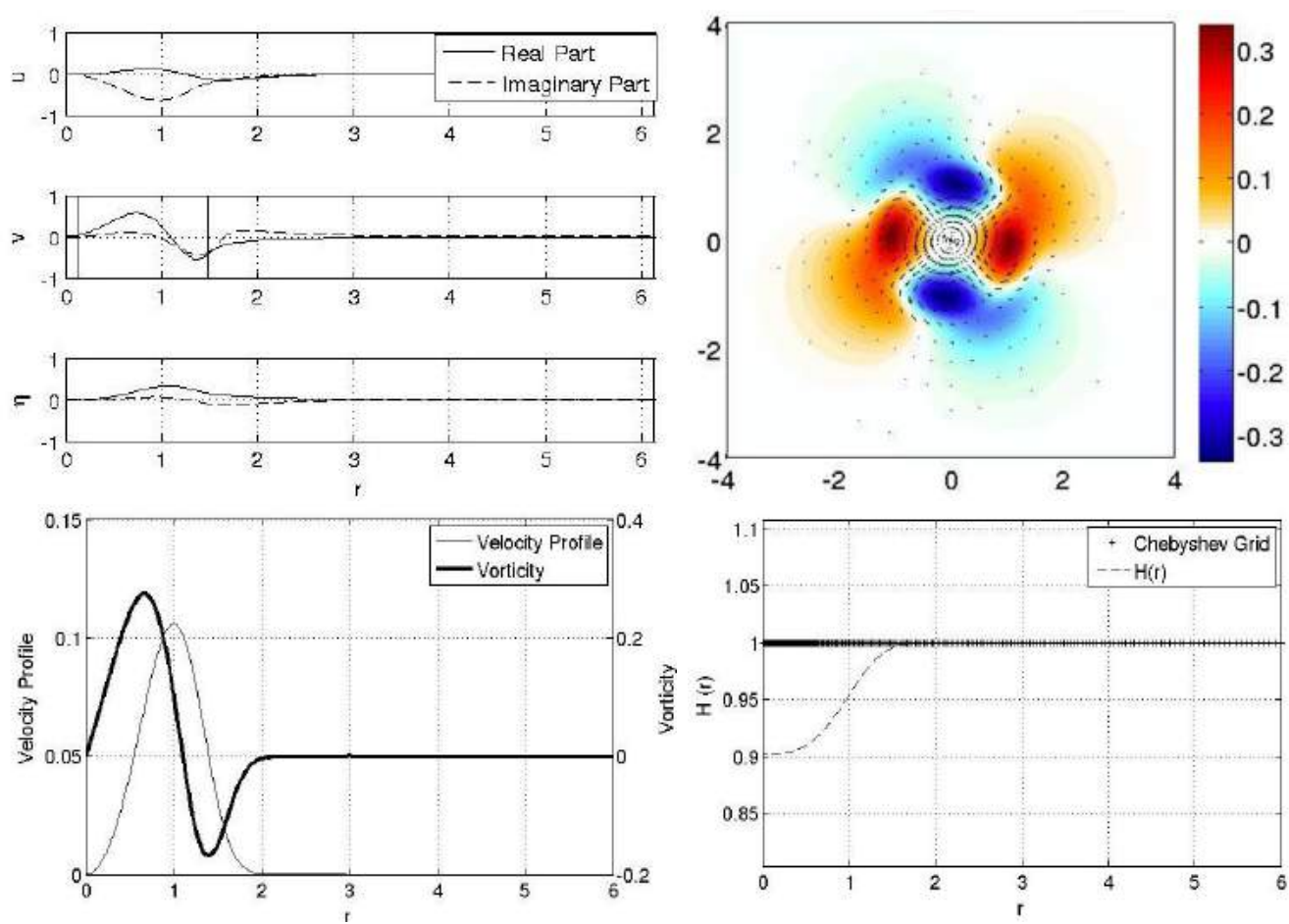

Figure 1. Upper row: left - radial structure $(u, v, \eta)(r)$ of the most unstable mode (vertical line: position of the critical level); right - corresponding pressure and velocity fields on the $x-y$ plane. Lower row: left - azimuthal velocity profile and vorticity of the background barotropic cyclone with $\alpha=4, \epsilon=0.1061$; right - Chebyshev grid points (resolution $N=550$ ), and thickness of the vortex $H(r)$. Stability analysis is performed within the "dry" model.
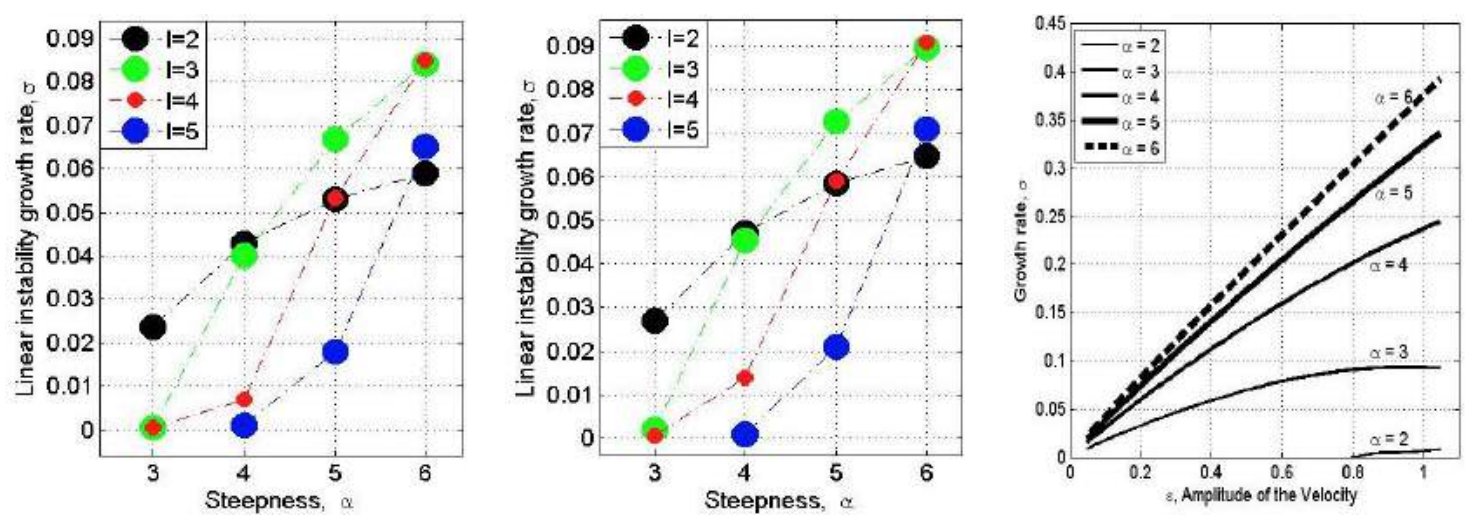

Figure 2. Dependence of the "dry" linear growth rates of different azimuthal modes on steepness for anticyclonic (left panel) and cyclonic (middle panel) vortices with $\epsilon=0.1414$, and dependence of the growth rate of the mode $l=2$ upon steepness and amplitude parameters $\alpha$ and $\epsilon$ (right panel)

\subsection{Linear stability of vortices in the two-layer model}

\subsubsection{Formulation of the linear stability problem}

The linearisation procedure around a vortex solution $H_{1}^{*}, H_{2}^{*}, V_{1}^{*}$ and $V_{2}^{*}$ in layer-wise cyclo-geostrophic equilibrium (14) in the two-layer case follows the same lines as in the onelayer case above. The eigen-frequencies and corresponding eigen-modes are found from the 

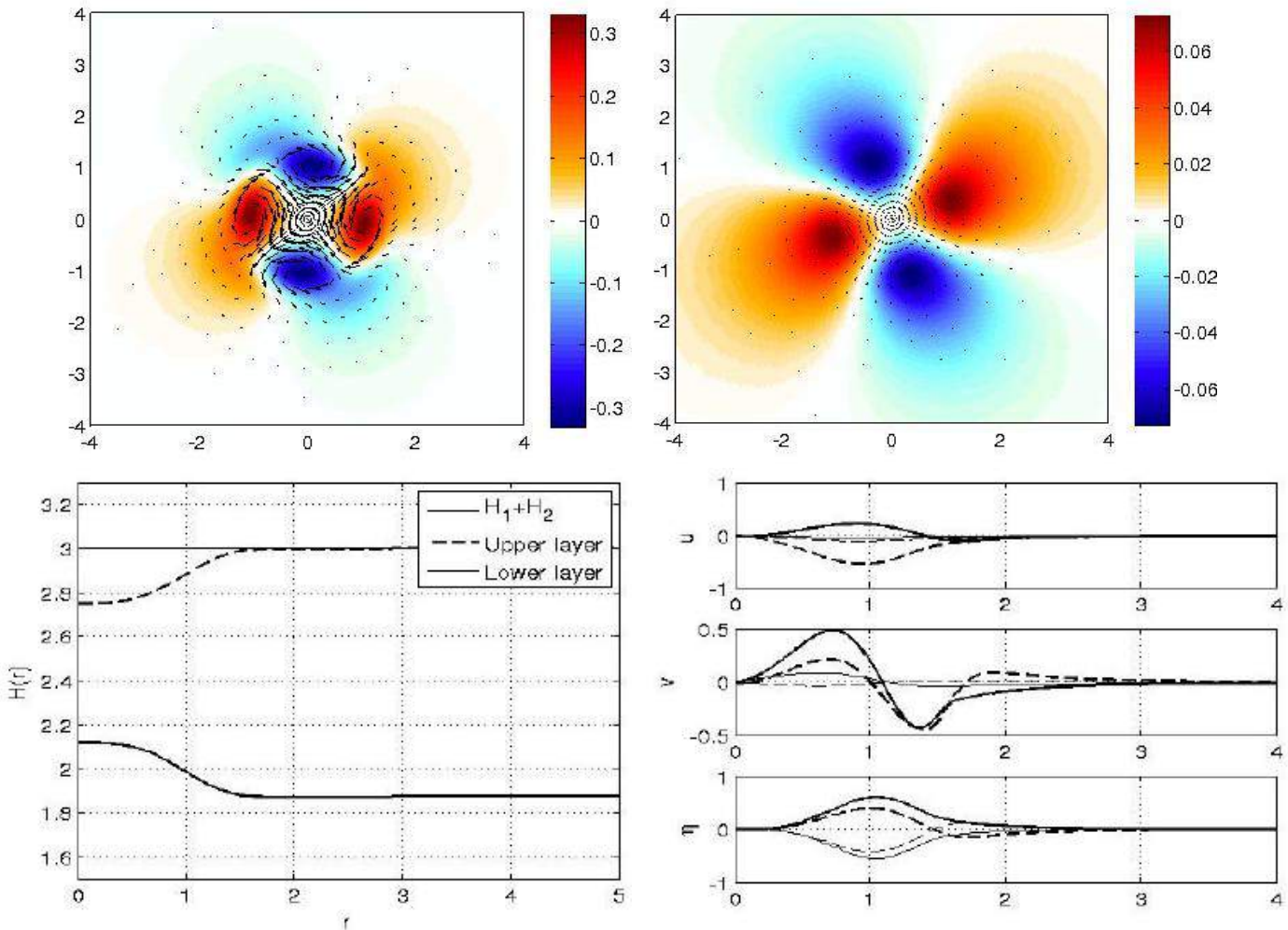

Figure 3. Structure of the most unstable mode of an upper-layer cyclone with $\alpha=4,, H_{2} / H_{1}=0.6, l=2, \epsilon=0.08$ and $s=1.37$. Upper row: left (right) - pressure and velocity fields in the $x-y$ plane in the upper (lower) layer Lower row: left $-H_{1}$ and $H_{2}$ of the background vortex, right - radial structure of the unstable mode, dashed (solid) lines: imaginary (real) part. Stability analysis is performed within the "dry" model.

non-dimensional eigen-problem:

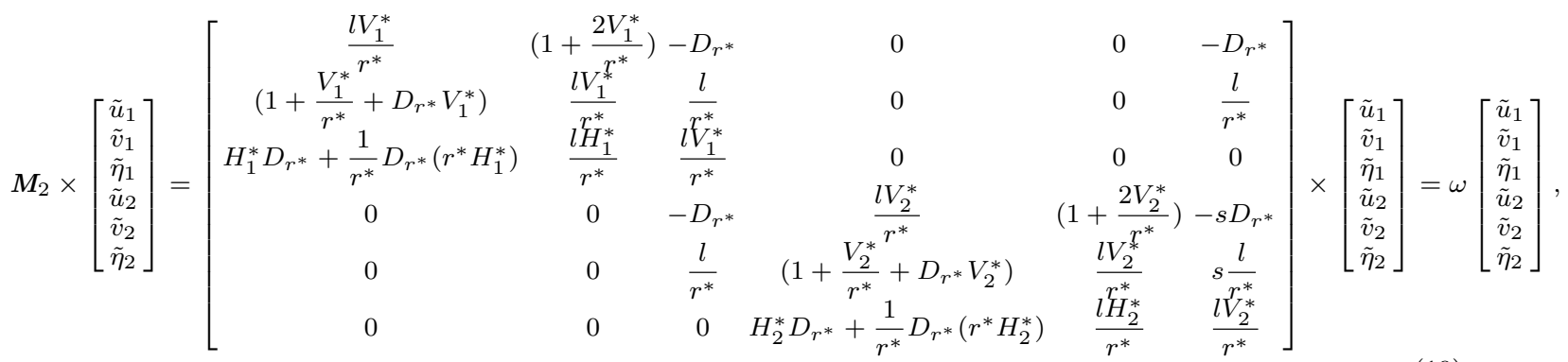

where $\boldsymbol{M}_{2}$ is the $6 \times 6$ operator matrix which can be rewritten in terms of Rossby and Burger numbers with the help of the scaling $r=L r^{*}$ similar to (18) (not shown).

\subsubsection{Results of the linear stability analysis}

As was already explained, we are interested in upper-layer cyclones. The most unstable mode of an $\alpha$-Gaussian upper-layer cyclone is presented in Figure 3. As in the one-layer case, we analysed the dependence of the growth rate on the parameters of the background vortex which is presented in Figs. 4 and 5 . An important conclusion following from this analysis is that there is a swap between $l=2$ and $l=3$ in azimuthal structure of the most unstable mode with changing steepness of the vortex profile and/or thickness ratio, for strong enough stratifications. 


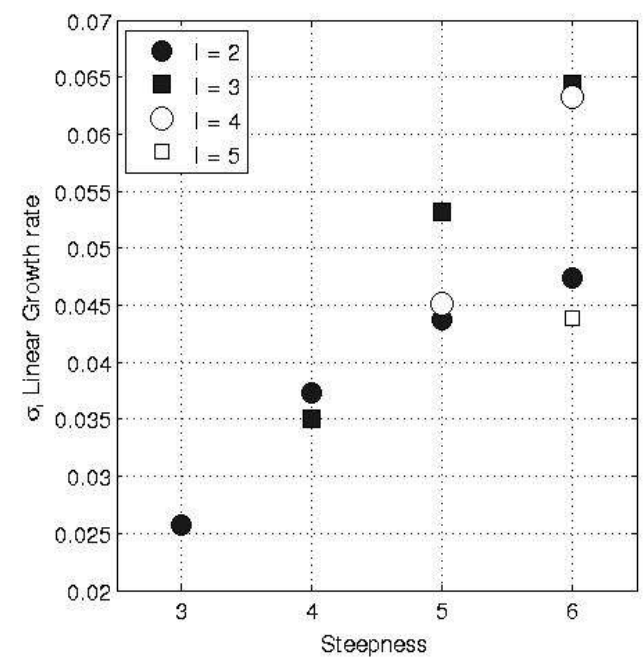

Figure 4. Dependence of the "dry" growth rates of different azimuthal modes on steepness for upper-layer cyclone with $\epsilon=0.1, H_{2} / H_{1}=0.6$, and $s=1.37$.
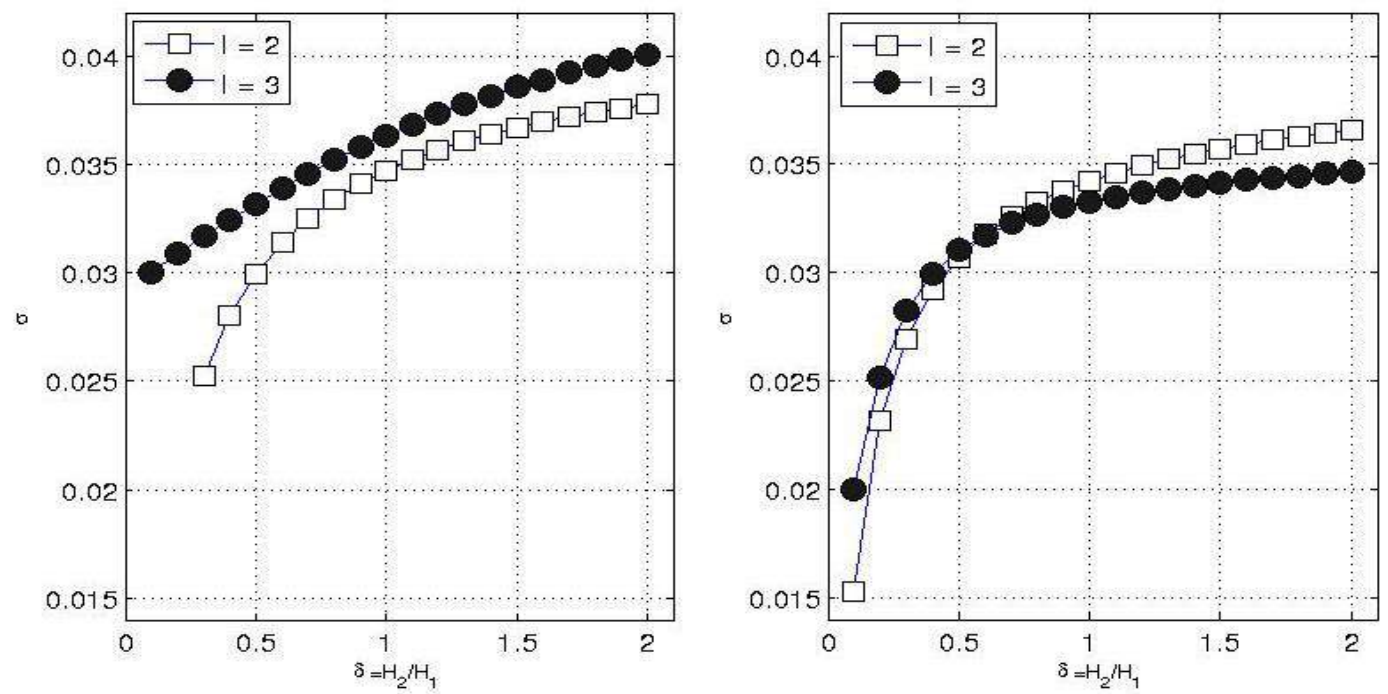

Figure 5. Variation of the "dry" growth rate of the most unstable mode of the upper-layer cyclone with $H_{2} / H_{1}$ and $\alpha=4$ for weak $(s=1.1$, left panel) and strong ( $s=1.37$, right panel) stratification.

4. Nonlinear evolution of barotropic and baroclinic instabilities of vortices: comparison of "dry", moist-precipitating, and moist-precipitating and evaporating simulations

In this section we present the results of comparison of nonlinear evolution of the barotropic and baroclinic instabilities of vortices which were analysed at the linear level in section 3 . We perform fully nonlinear numerical simulations in one- and two-layer mcRSW models with the help of the finite-volume well-balanced RSW code with a relaxation scheme for precipitation, as in (Lambaerts et al. 2011b, 2012). The simulations which are presented below are initialised, for both cyclonic and anticyclonic vortices, by the $\alpha$-Gaussian profiles of $H(r)$ and $V(r)$. The most unstable mode, which was obtained by the linear stability analysis of section 3 , was superimposed, with a small amplitude $\approx 0.01$, on the background vortex at the initial moment. A change of parameter $\beta$ can be absorbed in rescaling of $Q$ and $\tau$, so it was kept equal to unity in the simulations. $\tau$ was taken to be as short as possible, several time-steps, 
first, to be consistent with physical reality, and second, in order to be able to confront the results with qualitative analysis available in the immediate relaxation limit. $Q$ was chosen to be sufficiently close to $Q_{s}$, in order to assure non-negligible condensation. We will compare nonlinear evolutions of $l=2$ and $l=3$ instabilities and see that they are significantly different.

The following numerical experiments were performed:

- One-layer barotropic model: evolution of 1) anicyclonic and 2) cyclonic vortex with superimposed most unstable modes in dry (M), moist-precipitating (MP) and moistprecipitating with surface evaporation (MPE) configurations. All three surface evaporation schemes $E 1, E 2$, and $E 3$ were tested. The MPE III results are not systematically presented as with the above choice of initial (uniform) distribution of moisture they are similar to MPE II results.

- Two-layer baroclinic model: evolution of an upper-layer cyclone with superposed unstable modes with 1) dipolar and 2) tripolar azimuthal structure in all configurations mentioned above.

Pressure, velocity and PV fields obtained in these experiments were used for diagnostics of vortices, and divergence field was used to diagnose inertia-gravity waves. Humidity field $Q$ and precipitation field $P$ were used to diagnose evolution of moisture and condensation. The growth rates of the instability were analyzed with the help of dry energy norm of the perturbations.

\subsection{Evolution of the barotropic instability: dipolar splitting}

In this section we present results of numerical simulations of the evolution of the vortex instability in the barotropic one-layer model where the leading instability has azimuthal wavenumber $l=2$. Various diagnostics of the influence of moist effects upon developing instability are displayed.

\subsubsection{Vortex evolution as seen in the $P V$ and humidity field}

The inter-comparison of the development of the barotropic instability in "dry" (M), with moisture behaving as passive scalar, moist-precipitating (MP), and most precipitating and evaporating (MPE I, MPE II), with the surface evaporation parameterisations E1 and E2 discussed in section 2, environments are presented in Figs. 6 and 7, for anticyclonic and cyclonic vortices, respectively. The dry PV anomaly (PVA) $q-f / H_{0}$ is used for diagnostics. We also made experiments with the mixed parameterisation of evaporation E3 which give similar to E2 results (not shown). We will come back to this point later.

To analyse the results, let us first concentrate on the "dry" evolution of the instability (first row in Figs. 6, 7). For both anticyclone and cyclone it consists in appearance of satellite vortices of the sign opposite to the main vortex at the periphery of this latter. As time goes on, the core of the initial vortex becomes elliptic and two satellite vortices intensify. The satellite vortices exert shear and strain on the core, and finally split it in two. The two vortices originating from the core pair with satellites and produce two vortex dipoles running in opposite directions. This is a barotropic dipolar breaking described in the literature (Baey and Carton 2002).

Let us now see how precipitation and evaporation modify the "dry" evolution scenario. The initial moisture in all simulations was distributed uniformly and unsaturated, albeit close to saturation: $Q_{0}=0.89$, and $Q^{s}=0.9$. When $Q>Q^{s}$, as follows from (2) the precipitation switches on. As shown in section 2.1, precipitation and evaporation affect the PV. A useful insight on the evolution of vorticity in the presence of precipitation can be obtained by considering (4) in QG approximation. Following (Lambaerts et al. 2011b) we get: 

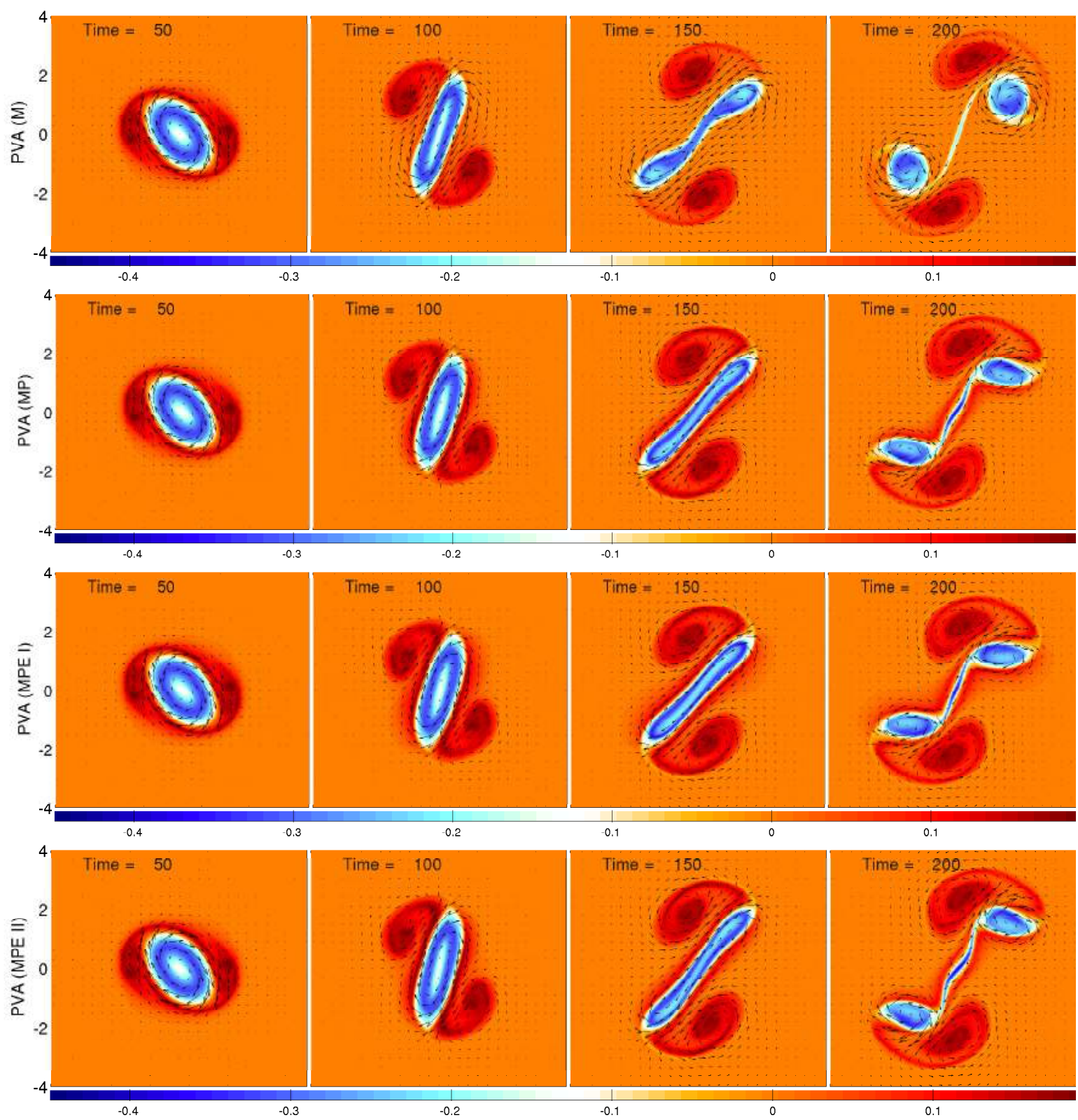

Figure 6. Evolution of PVA of the anticyclonic vortex with $\alpha=4, \epsilon=0.1061, l=2, \gamma=0.01, \delta=0.001$ during the saturation of the barotropic instability in four different environments: M, MP, MPE I, and MPE II, respectively from top to bottom.

$$
\left\{\begin{array}{l}
\left(\partial_{t}+\boldsymbol{v}_{g} \cdot \nabla\right)\left(\nabla^{2} \psi-\psi\right)=\beta P_{1} \\
\left(\partial_{t}+\boldsymbol{v}_{g} \cdot \nabla\right)\left(\tilde{Q}-Q^{s} \nabla^{2} \psi\right)=-P_{1}
\end{array}\right.
$$

where $\tilde{Q}$ is the moisture deficit with respect to the saturation, $\boldsymbol{v}_{g}$ is the geostrophic velocity, and $\psi$ is the geostrophic stream function of the flow $\boldsymbol{v}_{g}=\hat{z} \wedge \psi$. In the immediate relaxation limit $(\tau \rightarrow 0)$, we can assume that $\tilde{Q} \approx 0$ and get:

$$
Q^{s}\left(\partial_{t}+\boldsymbol{v}_{g} \cdot \nabla\right) \zeta_{g}=P_{1},
$$

where $\zeta_{g}=\nabla^{2} \psi$ is the quasi-geostrophic vorticity. It is clear from this formula that cyclonic motions in the precipitation regions become intensified. This is exactly what is observed in the second rows of Figs. 6, 7. As a consequence, the resulting dipoles become asymmetric, with a 

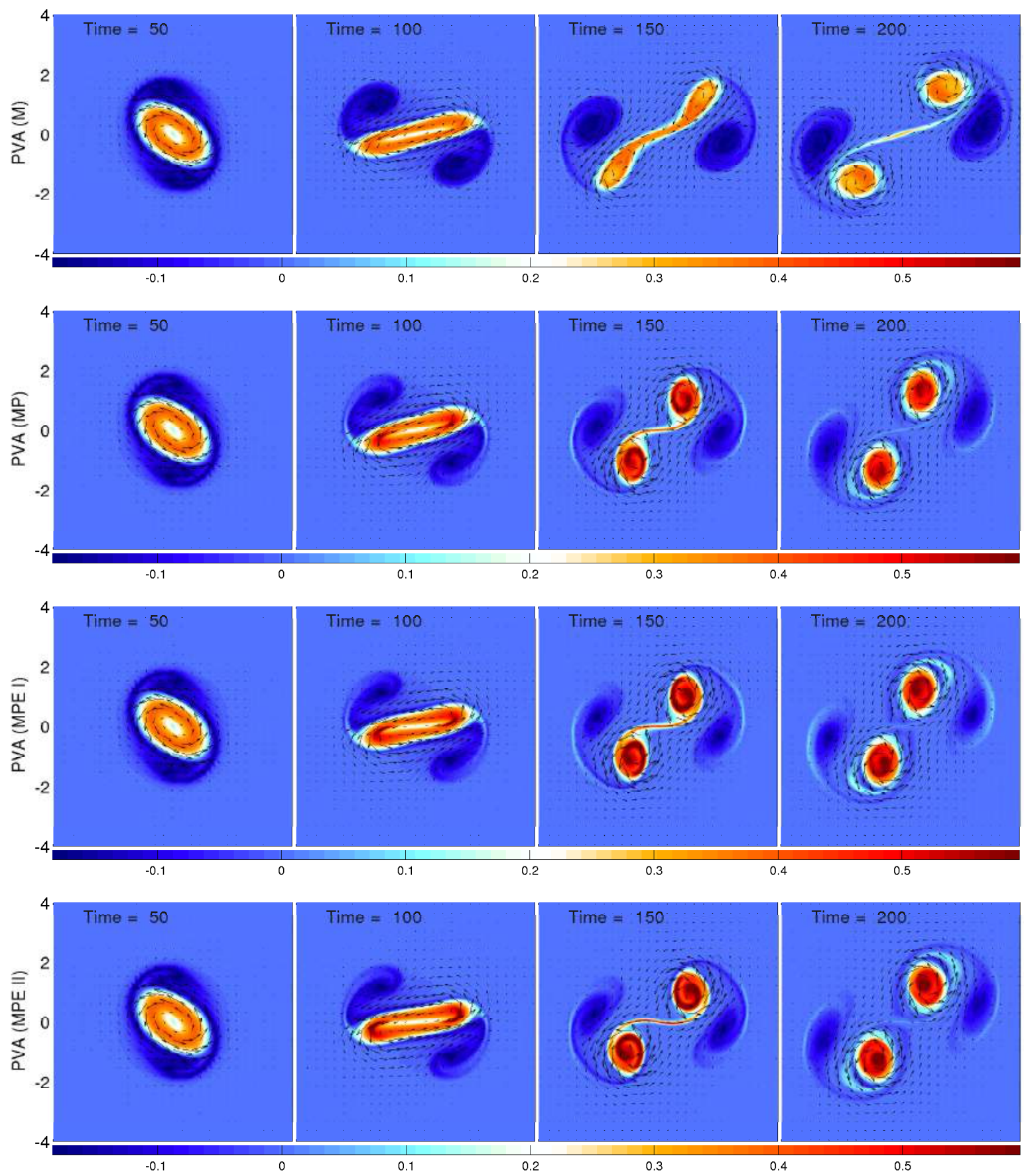

Figure 7. Same as in Fig. 6 but for the cyclonic vortex.

dominant cyclonic part. Correspondingly, their trajectories change and become meandering, as compared to the quasi-rectilinear ones in the dry case (not shown). These phenomena are pronounced even more in the presence of evaporation. For both parameterisations of evaporation, the cyclonic partner of each dipole pair is enhanced and occupies a larger area, while the anticyclonic partner is inhibited and shrinks, as compared with the case without evaporation. A net enhancement of the cyclonic component of the dipoles is confirmed by Fig. 8 where we show the corresponding evolution of maximum and minimum pressure. As follows from Fig. 8, in all precipitating environments the pressure maximum is weakened and 

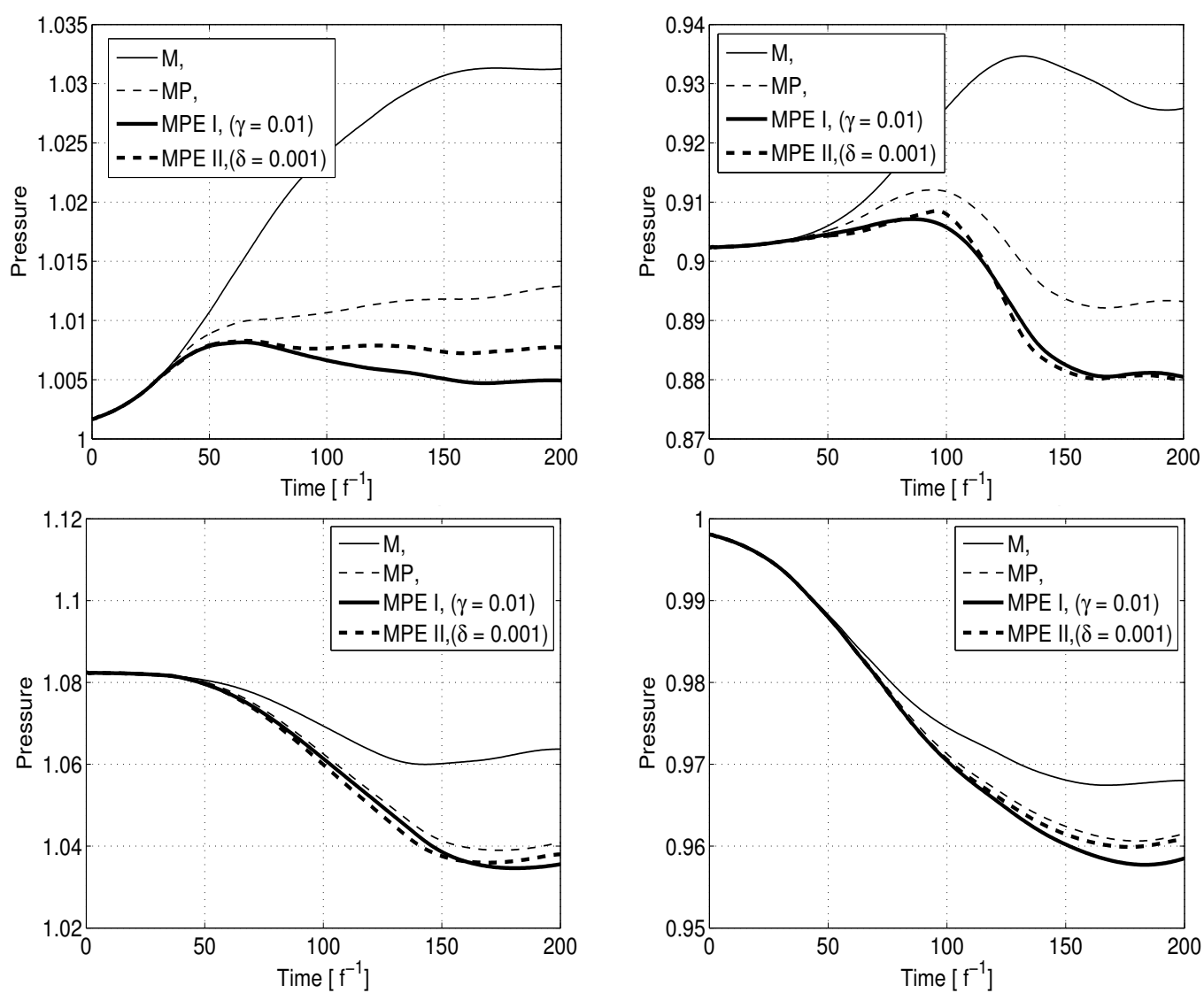

Figure 8. Evolution of the maximum (left) and minimum (right) pressure in different environments of the cyclonic (top), and the anticyclonic (bottom) vortices, both with $\alpha=4, l=2, \epsilon=0.1016$.

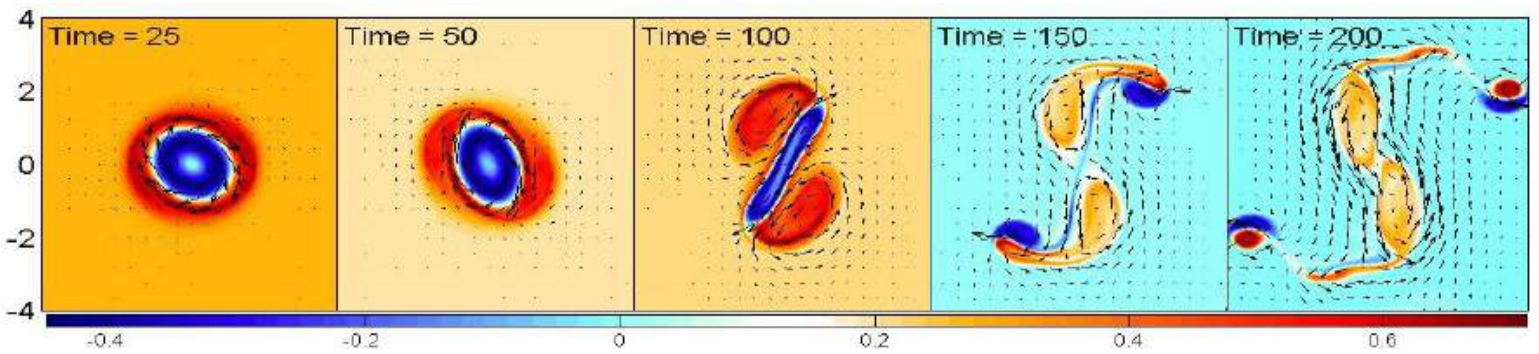

Figure 9. Evolution of PVA of anticyclonic vortex with $\alpha=4, \epsilon=0.1016$ in the MPE II environment. The surface evaporation coefficient $\delta=0.01$ is ten times larger than in the bottom row of Fig. 6

the pressure minimum deepened, as compared to the "dry" case, in full accordance with the PVA evolution.

It must be emphasised that the values of parameters $\gamma$ and $\delta$ have significant influence on the PVA evolution. Fig. 9 shows that enhancement of cyclonic vorticity is so strong at large values of evaporation coefficient $(\delta=0.01)$ that only a part of it enters the secondary dipole, which becomes very compact, while another part is shed forming a vorticity strip subject to secondary shear instabilities.

Comparison of evolution of humidity during the evolution of the instability of an anticyclonic vortex in "dry" and moist-precipitating environments is presented in Fig. 10. The areas of enhanced humidity, exceeding the saturation level, in the non-precipitating (M) simulation indicate locations of precipitation zones in the precipitating environment. 


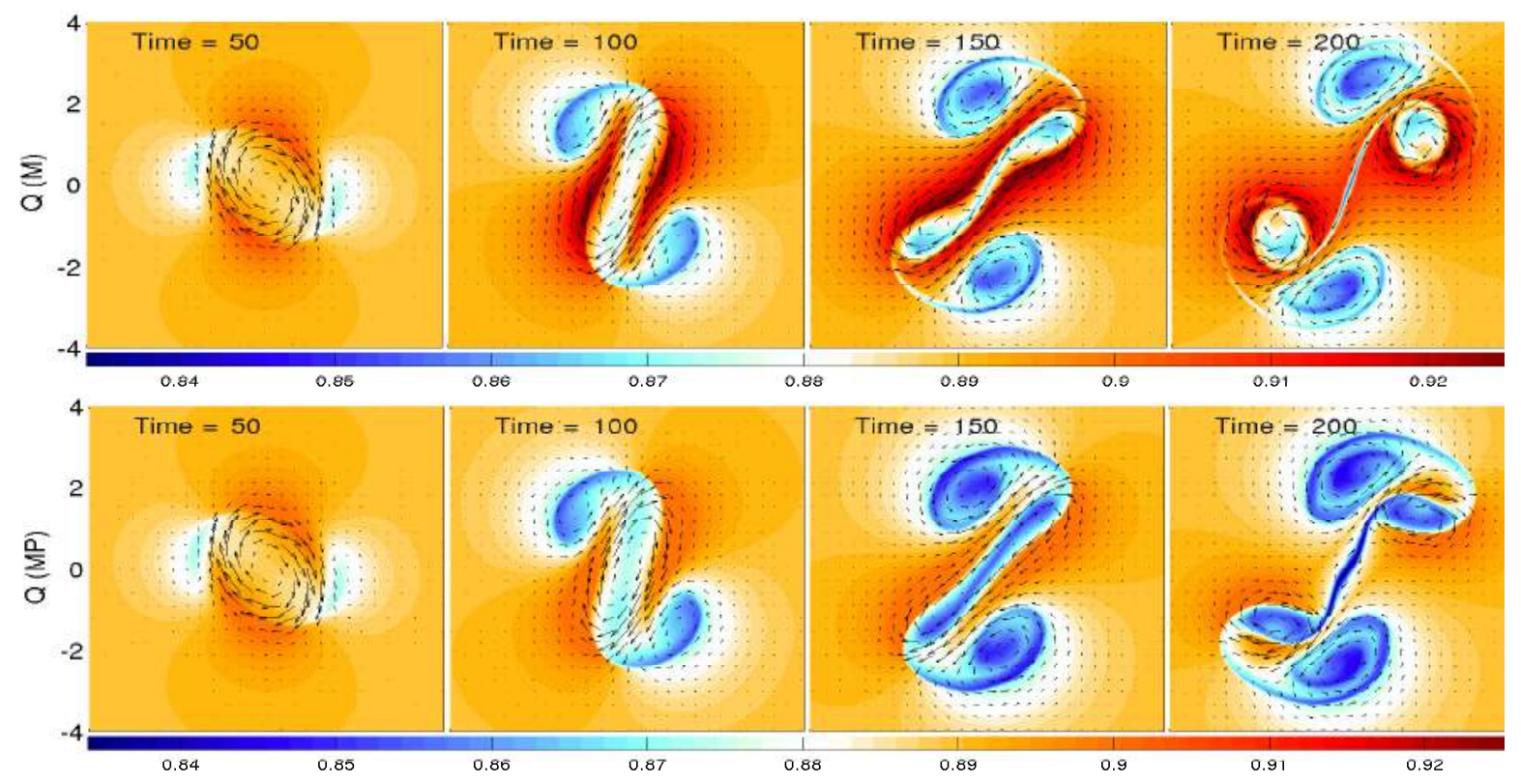

Figure 10. Evolution of the bulk humidity $Q$ in M (upper row) and MP (lower row) environments for the anticyclonic vortex with $\alpha=4, \epsilon=0.1061$.

\subsubsection{Quantifying evolution of the instability: growth rate and precipitation}

As was already said in section 2, condensation is an essentially nonlinear phenomenon, and thus the moist instability cannot be studied by the standard means of the linear stability analysis. To get an estimate of the growth rate of the moist instability we follow (Lambaerts et al. 2011b) and measure it in the simulations by using the dry energy norm:

$$
S^{2}(t)=\iint\left[H_{0}(x, y) \frac{u^{2}+v^{2}}{2}+g \frac{\eta^{2}}{2}\right], \quad \sigma(t)=\frac{1}{2} \frac{d}{d t} \log \left[\frac{S^{2}(t)}{S^{2}\left(t_{0}\right)}\right] .
$$

Linearisation of the "dry" system (4) about the state of rest leads to

$$
\frac{d S^{2}(t)}{d t}=-g H_{0}(x, y) \iint \nabla \cdot(\eta \boldsymbol{v}) d x d y-g \beta \iint \eta P d x d y
$$

The influence of precipitation is given by the second term in the r.h.s. of this equations, which shows that $s^{2}(t)$ is sensitive to the sign of the pressure perturbation in the precipitating region, cf. (Gill 1982, Lapeyre and Held 2004, Lambaerts et al. 2011b).

Fig. 11 represents the variation of the growth rate in time for both anticyclonic and cyclonic vortices, as follows from the simulations of Figs. 6, 7in different environments. The prediction for the "dry" growth rate obtained in section 3 is also shown for comparison. As follows from the Figure, at the very beginning $\sigma$ differs from the growth rate given by the linear stability analysis. The reasons for such behaviour are (1) discretization errors (2) the fact that the amplitude of the perturbation is small, yet finite, and thus the vortex with the superimposed perturbation is (slightly) unbalanced and undergoes geostrophic adjustment (Zeitlin 2008) (see also section 4.1 .3 below). It takes several inertial periods to reach the geostrophic balance. After this initial adjustment, the growth rate for about $15 f^{-1}$ stays close to the linear one. (The difference between the theoretical and measured growth rates diminishes with increasing resolution and computational cost - not shown). Then the instability starts to dynamically saturate and the growth rate diminishes. At the onset of the precipitation $\sigma$ experiences a short transient increase in precipitating environments, which is much more pronounced for the cyclone, and then steadily decreases. This decrease is substantially faster in precipitating 

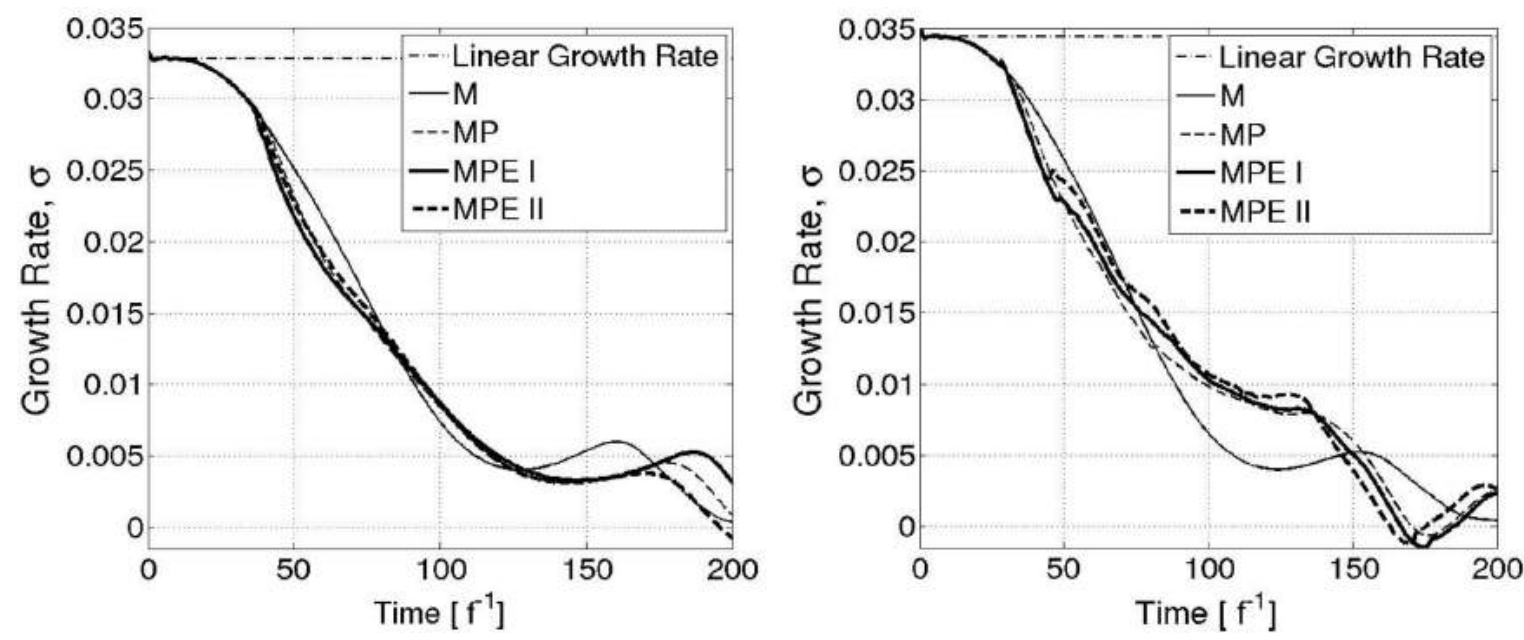

Figure 11. Variation of the growth rate of the barotropic instability of cyclonic (left) and anticyclonic (right) vortices, both with $\alpha=4, \epsilon=0.1061, l=2, \gamma=0.01$ and $\delta=0.001$.

environments, as compared with the "dry" one. Such reduction of the growth rate can be explained by the positive thickness anomaly in the precipitating region, cf. (23), which will be confirmed in the next subsection. It should be emphasised that observed behaviour differs compared to what was observed in the simulations of nonlinear evolution of the barotropic instability of a jet in (Lambaerts et al. 2011b), where a persistent increase of the growth rate of the instability was detected at the onset of precipitation. This means that the influence of condensation and related latent heat release upon the growth rate of the barotropic instability is not universal and depends on fine details of the flow - see also below.

In order to better understand the correspondence between the predictions of the linear stability theory of section 3 and the results of numerical simulations, we decomposed the pressure and velocity anomalies with respect to the unperturbed vortex in Fourier series in azimuthal wavenumber $l$ and traced the evolution of several Fourier-modes of the pressure field, as given by numerical simulations in the dry environment initialised with the $l=2$ perturbation. The results are displayed in Fig. 12 and are similar for the Fourier-modes of the velocity field (not shown). At initial stages $l=2$ mode is dominant and follows well the prediction of the linear theory. At the same time the mode $l=4$, and then other even modes, start to grow from their initially negligible, but non-zero due to discretisation errors, values. This growth is due to nonlinear interaction of the original $l=2$ perturbation, with itself, which projects onto the $2 l=4$ mode, but also with other modes, which leads to eventual dynamical saturation of the instability for both cyclonic and anticyclonic vortices. By symmetry reasons the amplitudes of the modes with odd $l$ remain very small. We were routinely making such benchmarks in all simulations and will not present them anymore.

Let us now quantify the precipitation during the evolution of the instability. We recall that in the immediate relaxation limit precipitation and wind divergence are directly linked, cf. (3). We checked that this is, indeed, the case (not shown). The evolution of the total amount of precipitation in the simulations of Figs. 6, 7 is presented in Fig. 13. As follows from the Figure, the evolution of precipitation is bell-shaped before the splitting of the initial vortex into two dipoles, and has a secondary peak due to precipitation inside the secondary dipoles. This peak is due to the fact that an increase of convergence, and hence the precipitation, is observed inside the anticyclonic partners of the secondary dipoles. This observation is confirmed by Fig. 14, where the evolution of the PVA and precipitation, as given by the simulation of Fig. 6, are superimposed. It is worth noting that precipitation patterns in all simulations are 

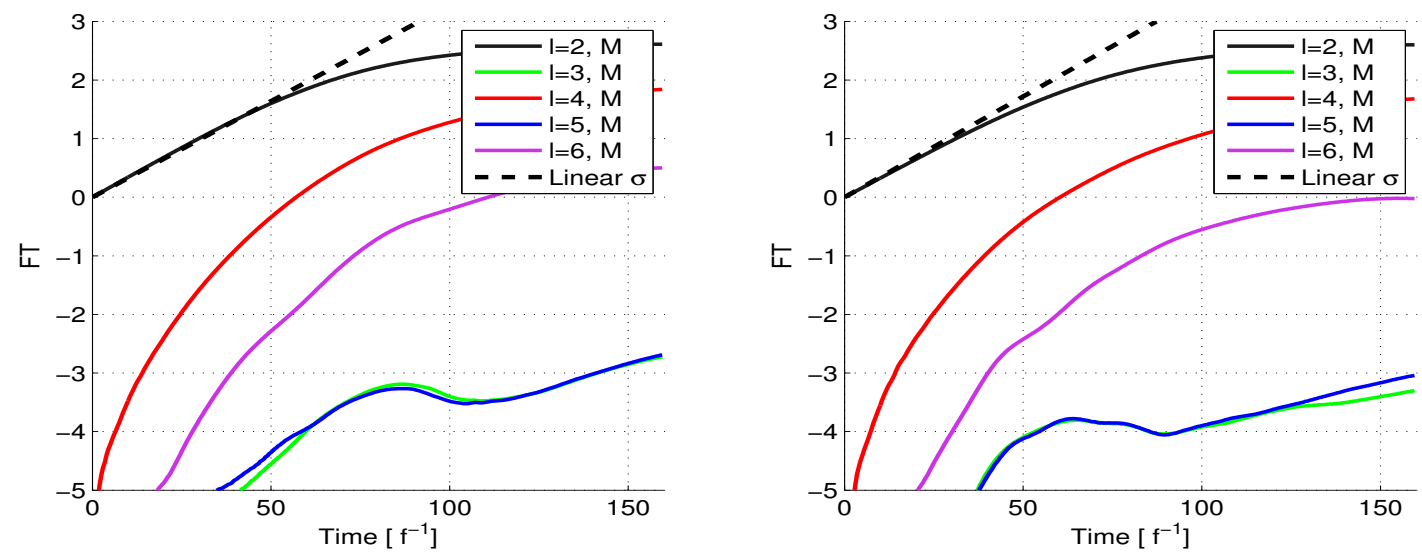

Figure 12. Logarithms of the normalised amplitudes of the first 6 Fourier modes of pressure as functions of time during the "dry" evolution of the barotropic instability of the cyclonic (left) and anticyclonic (right) vortices. $\alpha=4, l=2$, $\gamma=0.01$
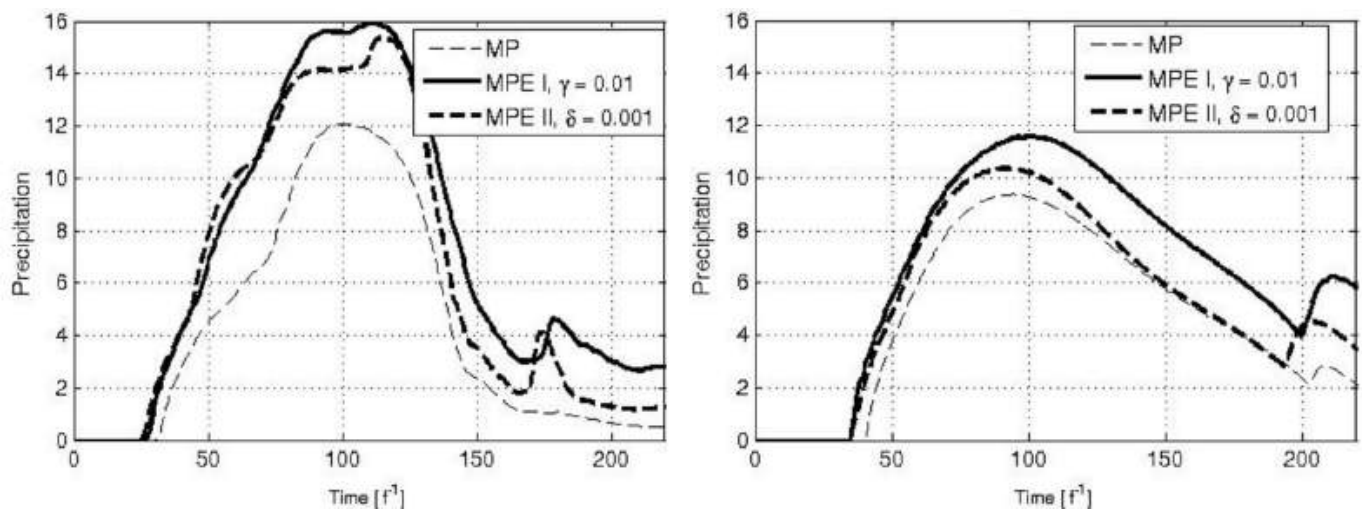

Figure 13. Total precipitation of the cyclonic (left panel) and anticyclonic (right panel) vortex, both with $\alpha=4, \epsilon=$ 0.1061 , in different environments.

close and do not depend much on the parameterisation of surface evaporation. The MPE II and MPE III patterns are practically identical. As is clear from Fig. 7, the intensity of the anticyclonic components of the secondary dipoles in the case of primary cyclone is much weaker than for the primary anticyclone, which explains the difference in precipitation patterns between the two. We finally present in Fig. 15 the evolution of the pressure anomaly $\eta$ with respect to initial vortex profile, with superposed precipitation, which corresponds to the (MP) simulation of Fig. 6. As follows from the figure, precipitation is correlated with positive values of $\eta$ which explains is destructive influence upon the growth rate, cf. 23.

\subsubsection{Inertia-gravity wave (IGW) emission}

As was already said in the Introduction, the question of IGW emission during the evolution of the instability, and of the influence of moist processes upon it, is of importance. We have analysed the IGW emission for cyclonic and anticyclonic vortices in all four environments. As a diagnostic of the IGW activity $W$ we calculated the modulus of wind divergence integrated over an annulus of non-dimensional width 0.5 situated sufficiently far from the vortex core, at the non-dimensional distance 5.5 from the center:

$$
W=\int_{r=5.5}^{r=6} r d r \int_{0}^{2 \pi} d \theta|\nabla \cdot \mathbf{v}|
$$

The time-evolution of thus defined IGW activity is presented in Fig. 16 for both anticyclonic 

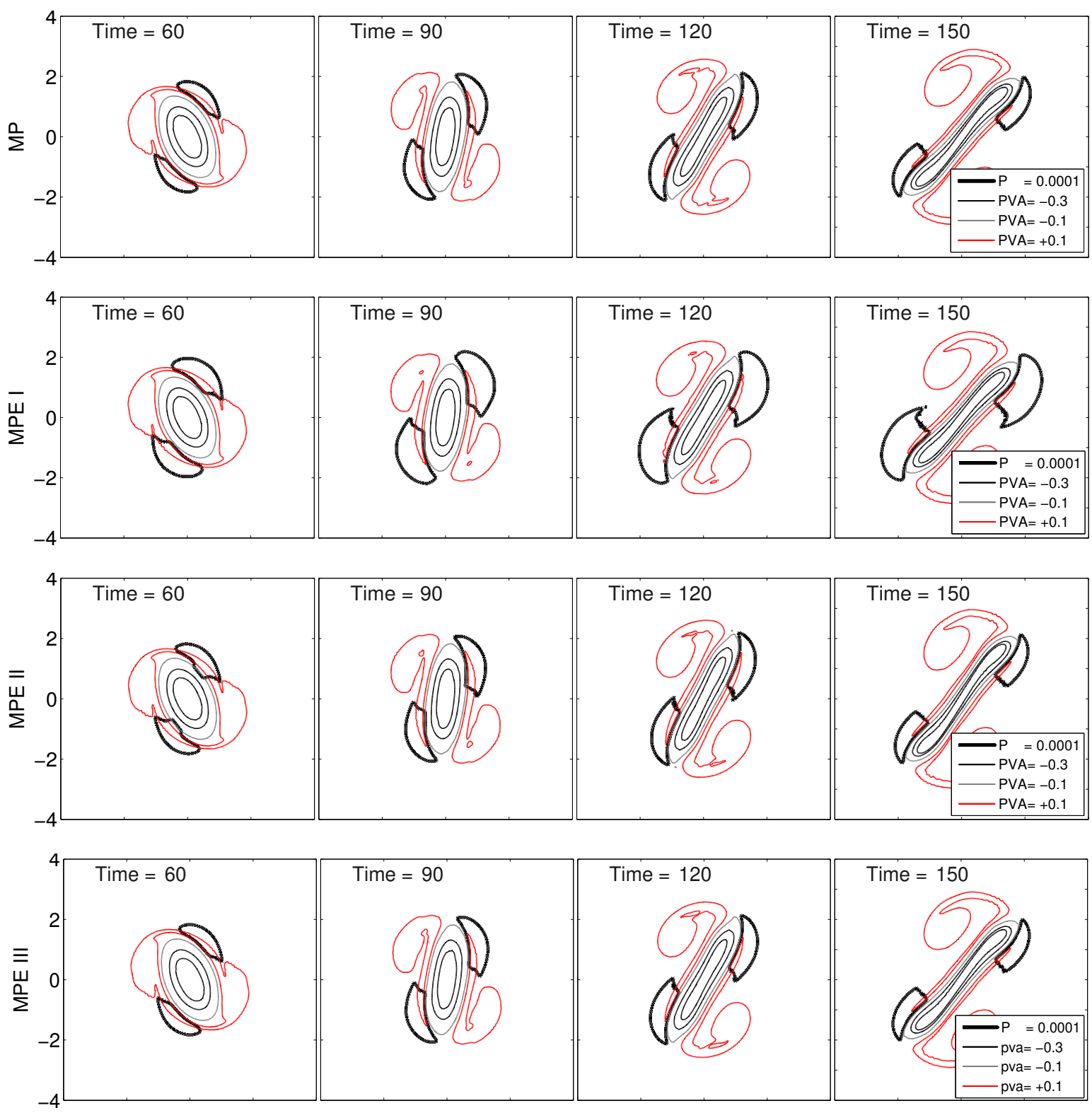

Figure 14. Joint evolution of PVA (thin contours) and precipitation (inside the thick contours), as follows from MP, MPE I, MPE II, and MPE III $(\kappa=0.05)$ simulations of Fig 6 respectively from top to bottom.

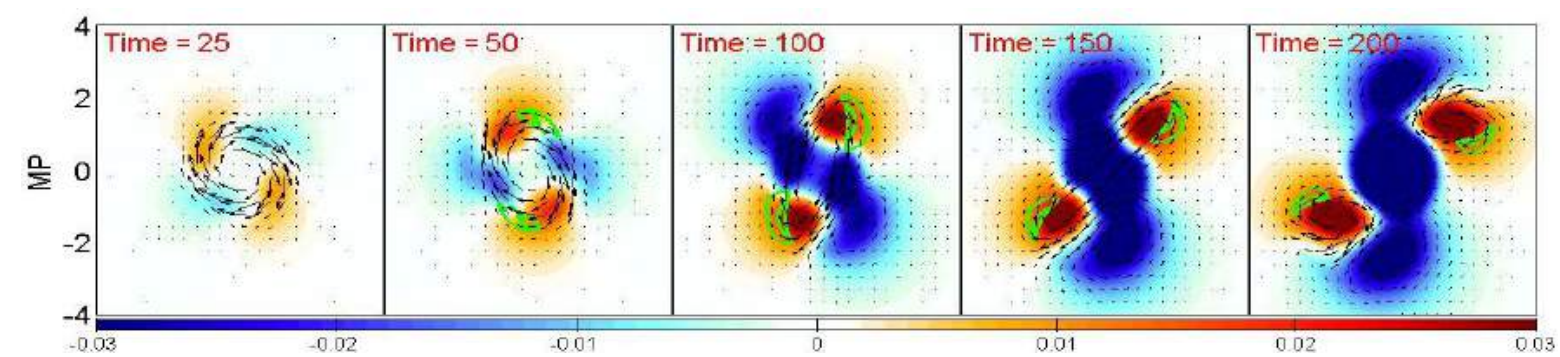

Figure 15. Joint evolution of pressure anomaly $\eta$ (colors) and precipitation (contours) in the MP environment for the anticyclonic vortex with $\alpha=4, \epsilon=0.1061$. 

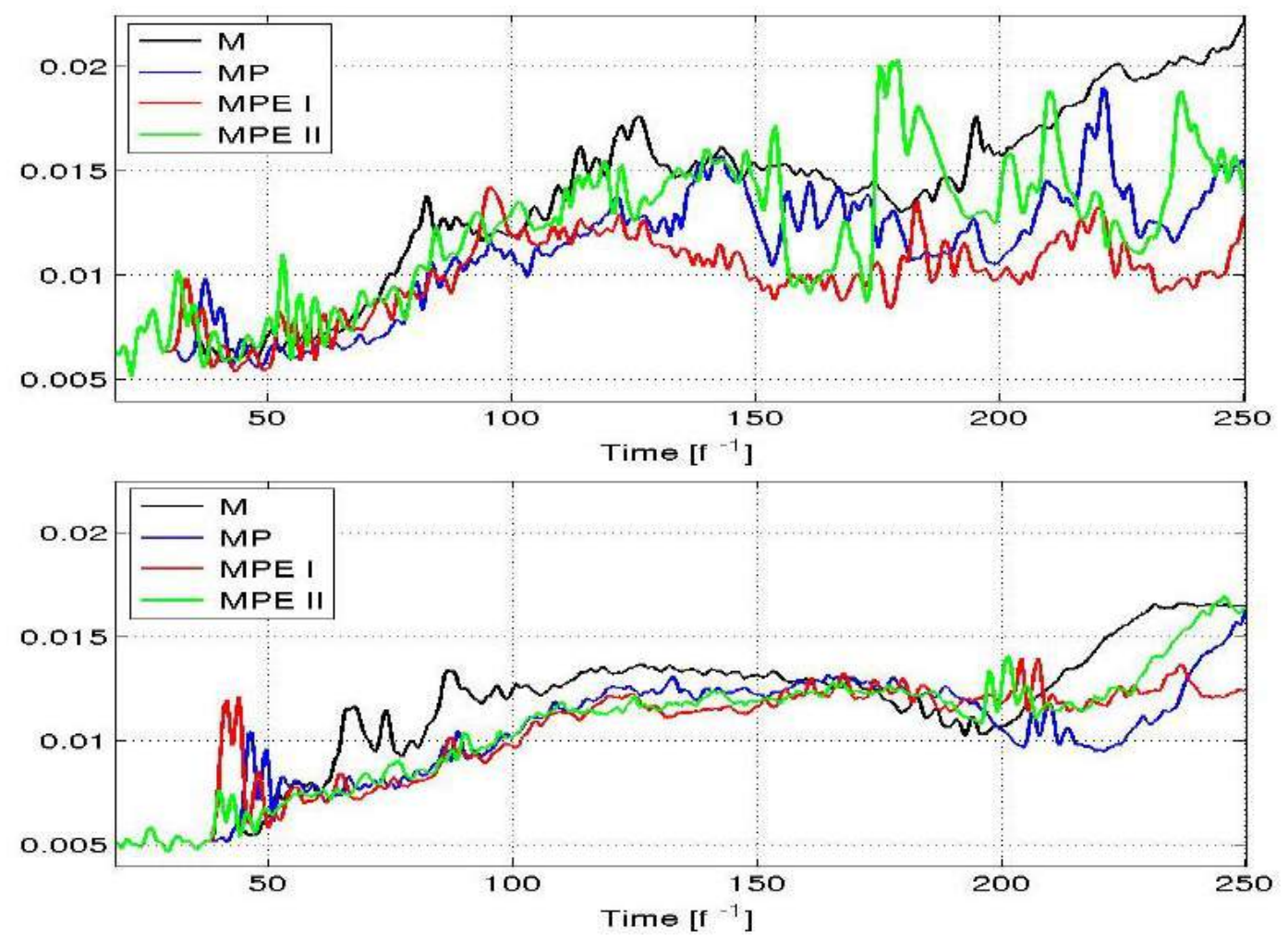

Figure 16. Evolution of the wave activity $W$ of the cyclonic (top) and anticyclonic (bottom) vortices with $\epsilon=0.1061, \alpha=$ $4, l=2$ during the evolution of the barotropic instability in four different environments.

and cyclonic vortices. The IGW emission starts in both cases early, together with the elongation of the vortex core. A notable increase in the wave activity takes place when the core vortex, accompanied by its two satellite vortices, splits into two dipoles at $t \approx 200$. Another remarkable increase in the wave activity happens at the beginning of the precipitation around $t=50$. The IGW emission has substantially stronger peaks in MP, MPE I and MPE II environments, as compared to the "dry" one M. The wave field corresponding to the events of strongest IGW emissions: (1) at the onset of precipitation, (2) after formation and separation of two dipoles is represented in the upper panel of Fig. 17. The fact that it is not only the reorganisation of the flow, but also moist effects which are at the origin of the increase in IGW activity, follows from inter-comparison of MP and MPE curves in Fig. 16, showing that with an increase of precipitation due to evaporation the IGW activity increases. Fig. 17 also shows a cyclone-anticyclone asymmetry in what concerns the wave emission, which is much more pronounced for the cyclone, starting from $t \approx 100 f^{-1}$. A strong maximum in wave activity produced by the cyclone in the MPE II environment corresponds to the high-amplitude wavepacket presented in the lower panel of Fig. 17. Yet, this large-amplitude IGW is not convection - coupled, except for locations where the wave is passing through the second vortex dipole, as follows from the distribution of precipitation which is not coupled to the wave.

\subsection{Evolution of the baroclinic instability: dipolar splitting}

For the analysis of the evolution of the baroclinic instability, we will be following the same lines as for the barotropic instability, except that we will be considering only a cyclonic vortex in the upper layer, as in Sec. 3.2 above. 

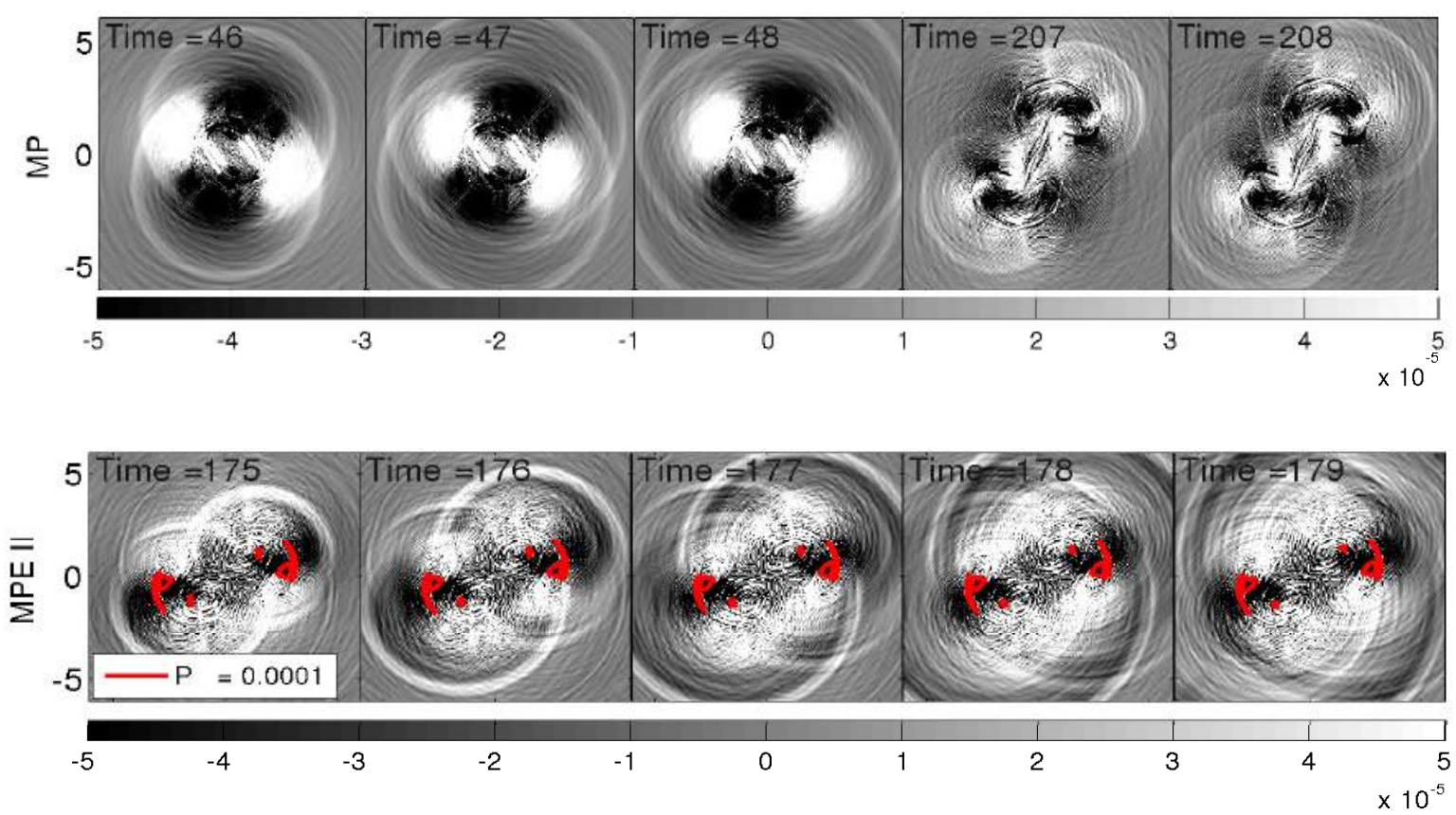

Figure 17. Inertia-gravity wave emission as seen in the divergence field: Upper row - anticyclonic vortex with $l=2, \epsilon=$ $0.1061, \alpha=4$ in MP environment at (1) the beginning of precipitation about $t=45 f^{-1}$ and (2) the separation of two dipoles about $t=207 f^{-1}$; Lower row - cyclonic vortex with $l=2, \epsilon=0.1061, \alpha=4$ in MPE II environment at $t \approx 175 f^{-1}$. Precipitation zones are delimited by thick contours.

\subsubsection{Vortex evolution as seen in the PV and humidity field}

We present in Figs. 18, 19 the evolution of the PVA, respectively in upper and lower layers, during the evolution of the baroclinic instability of the upper-layer cyclone. In the upper layer, the evolution is very similar to that of we have seen in the barotropic case. In the lower layer, the initial negative (due to the absence of low-layer velocity of the background vortex and a bump in thickness, cf. Fig. 33 PVA is stretched and positive values gradually appear forming secondary dipoles. Enhancement of positive PVA in all precipitating cases is spectacular. It leads to effective barotropisation of secondary dipoles.

\subsubsection{Quantifying development of the instability: growth rate, precipitation, and IGW emission}

As in the barotropic case, we calculate the growth rate based on the dry energy norm, which displays a similar behavior: following the linear stability analysis prediction for a dozen of inertial periods, and then decaying, with a transient increase at the stage of formation of lower-layer dipoles.

The precipitation peak in MP and MPE I environments corresponds to the process of splitting of the initial vortex into two dipoles. The continuing increase in precipitation in MPE II environment is related to a large value of evaporation coefficient and an increase of velocity in intensifying lower-layer dipoles. It should be emphasised that overall precipitation remains very small, as compared with the lower-layer vortex case of section 4.1.2. However, the position of precipitation areas with respect to the vortex is different, and their spread in the MPE II case is wider, as follows from Fig. 20.

The evolution of activity of baroclinic waves, calculated with the help of divergence of the baroclinic velocity field $v_{1}-v_{2}$ in the same way as in one-layer case, is presented in Fig. 21 . Apart from the initial peak which is related to the stretching of the core vortex, like in the 

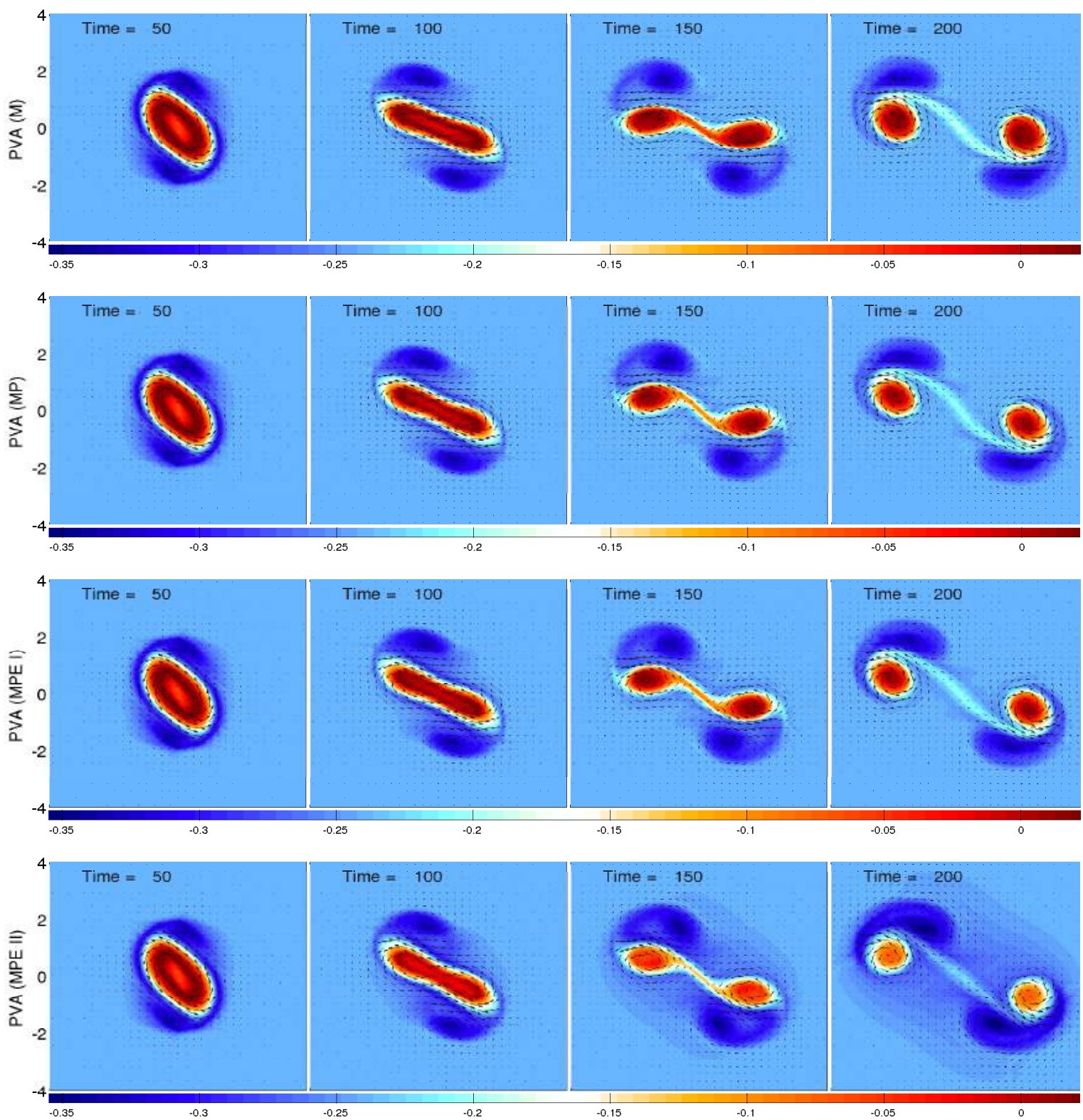

Figure 18. Evolution of PVA in the upper layer during the evolution of the $l=2$ instability of the upper-layer cyclone with $\alpha=4, \epsilon=0.08, s=1.37, H_{0}=3, H_{2} / H_{1}=0.6, \delta=0.05, \gamma=0.05$.

barotropic case, no secondary enhancement of the IGW activity is observed. This is due to the weak divergence field in the lower layer during the whole simulation, and weak precipitation.

\subsection{Nonlinear evolution of the baroclinic instability: tripole formation}

Let us now sketch what happens if the mode $l=3$ is the most unstable one. Following the same lines as in the previous case of the most unstable mode with $l=2$, we performed numerical simulations of developing instability in different environments. We again checked that the flow evolution follows the linear stability analysis at initial stages (not shown). We show the results for pressure evolution in the lower, most interesting, layer in Fig. 22.

Comparison of the evolution of the moisture content in "dry" and moist-precipitating sim- 

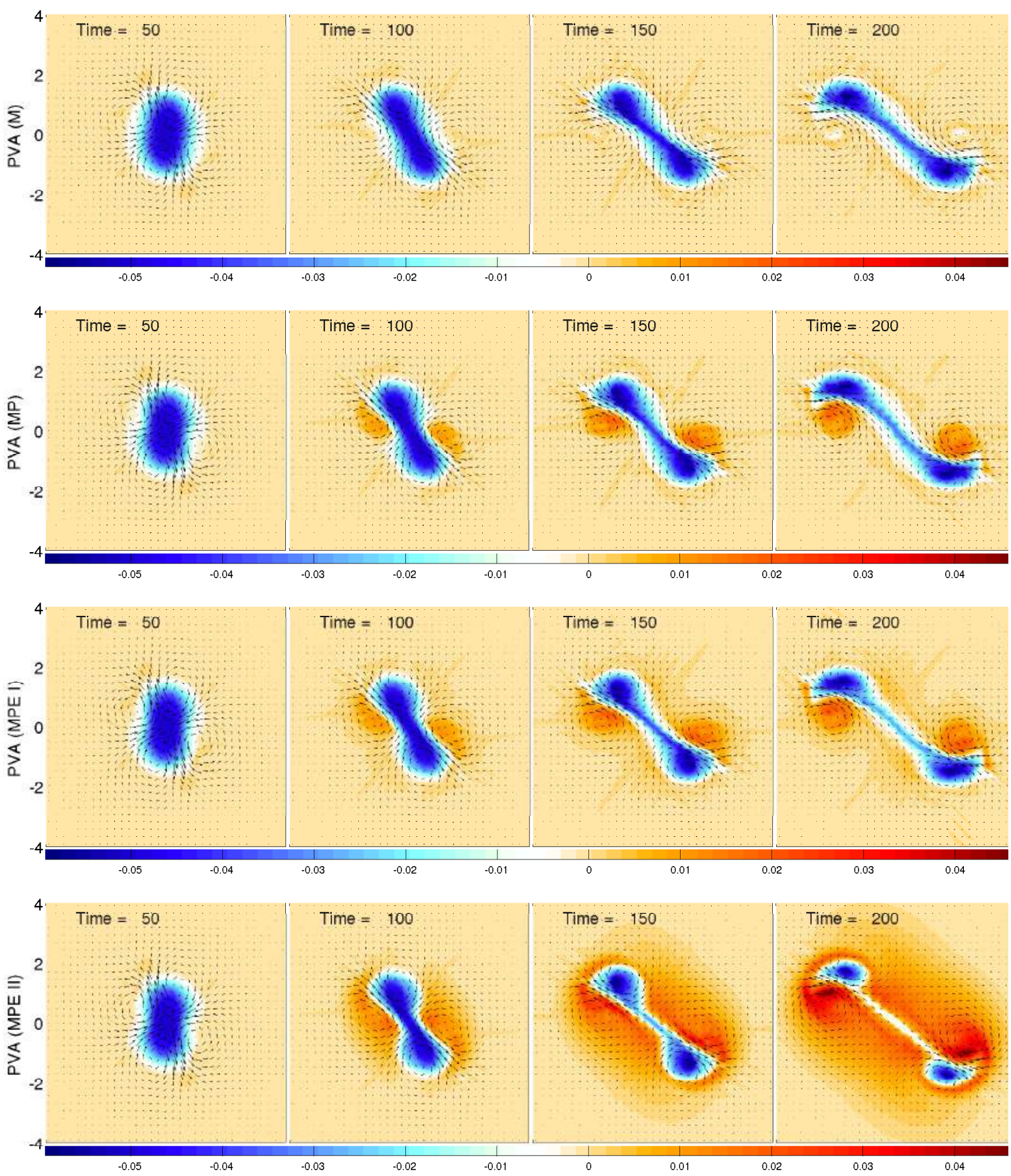

Figure 19. Same as in Fig. 18 but for the lower layer.

ulations is presented in Fig. 23. The upper row of this Figure clearly indicates precipitation zones appearing in precipitating environment in the middle row, which is confirmed by the bottom row, where joint evolution of PVA and precipitation in the lower layer is displayed. As follows from this Figure, the precipitation pattern is totally different compared to the dipolar breakdown case, with formation of characteristic rain bands. We observe considerable quantitative differences of tripolar evolution scenario, as compared to dipolar breakdown, in the behaviour of the growth rate and the wave activity. Evolution of the growth rate, calculated, as usual, with the help of the dry energy norm is presented in Fig. 24. 

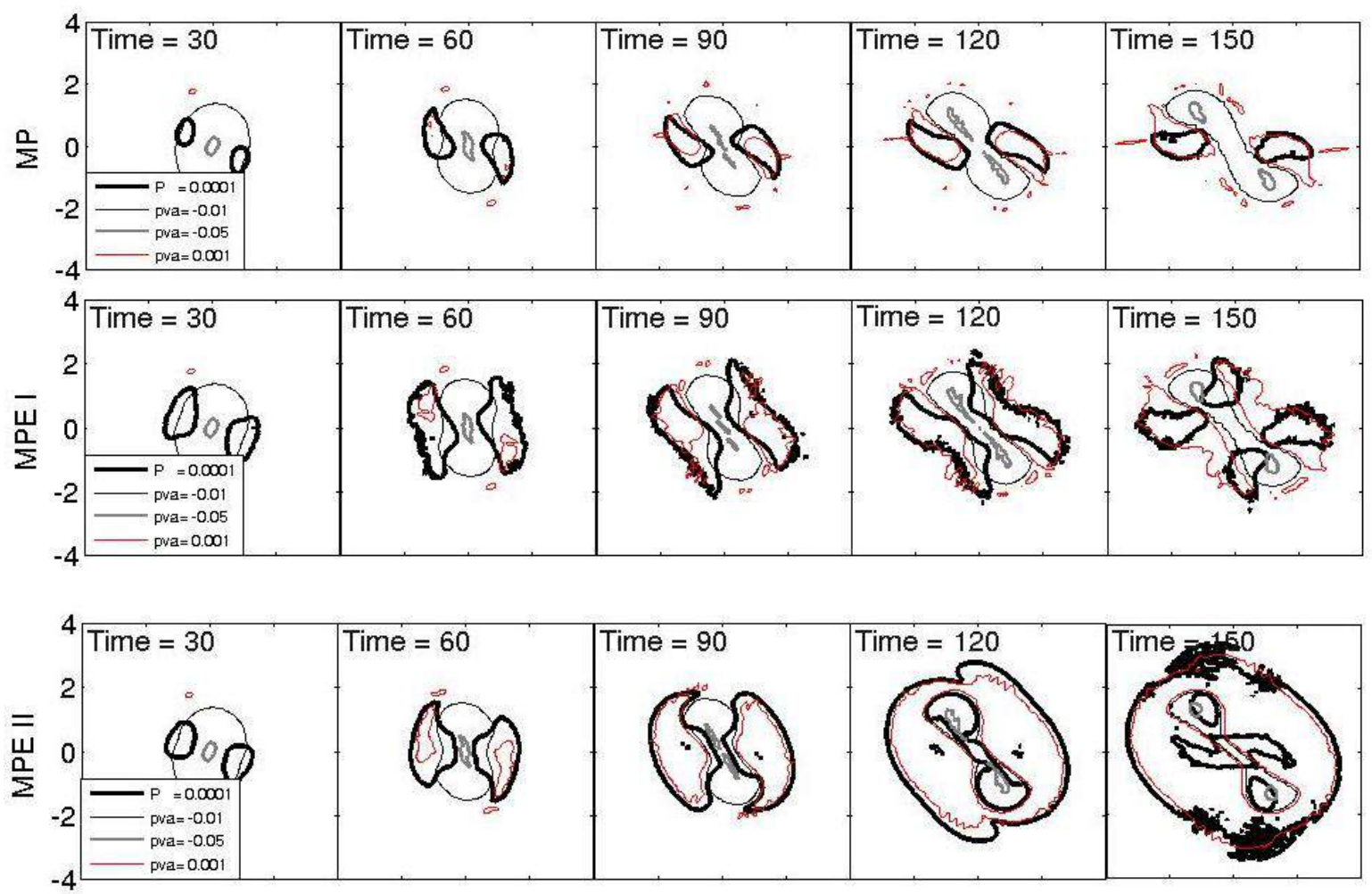

Figure 20. Superposition of the precipitation and PVA in the lower layer during dipolar splitting of upper-layer cyclone. Thick lines delimit precipitation regions. $\alpha=4, \epsilon=0.08, s=1.37, H_{0}=3, H_{2} / H_{1}=0.6, \delta=0.05, \gamma=0.05$.

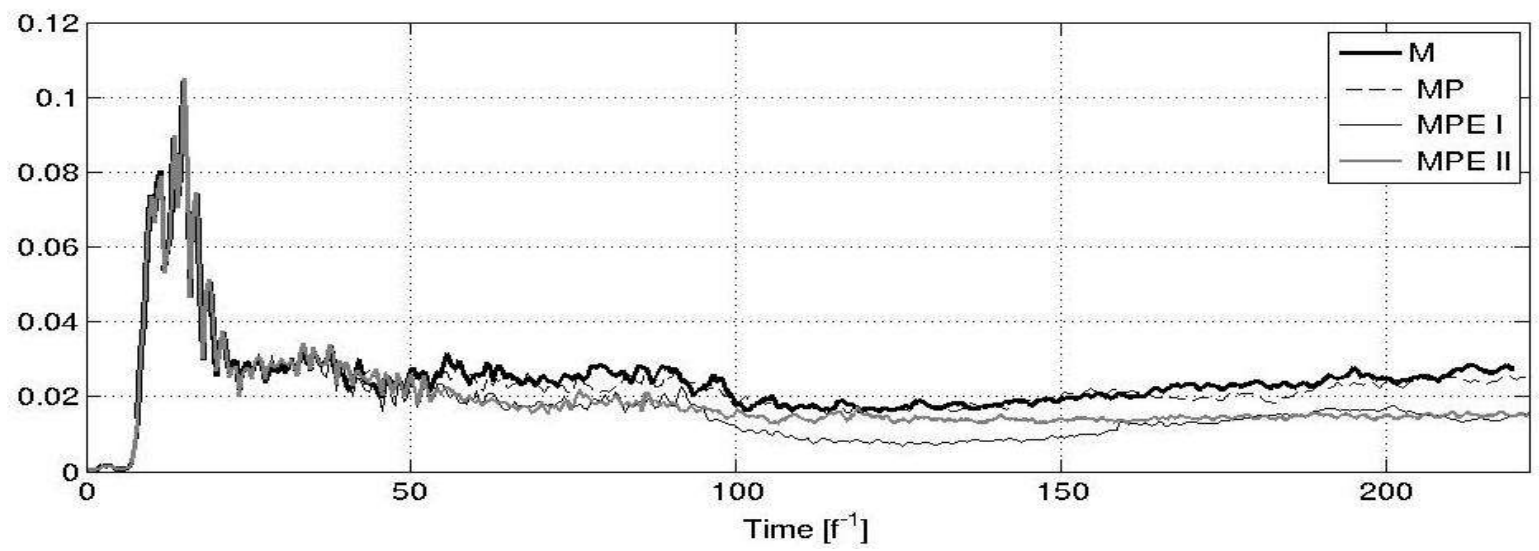

Figure 21. Evolution of the wave activity of baroclinic IGW $\left(\alpha=4, \epsilon=0.08, s=1.37, H_{0}=3, H_{2} / H_{1}=0.6, \delta=\right.$ $0.05, \gamma=0.05)$ during the evolution of the baroclinic instability of a vortex with $l=2$ most unstable mode.

It shows an enhancement of the growth rate, which becomes higher than the "dry" one due to precipitation. At the same time the dynamical saturation (decrease of the growth rate) is faster in the moist-precipitating environment than in the "dry" one. Such behaviour was not observed in the dipolar splitting scenarios, both barotropic and baroclinic, cf. Fig. 11. On the contrary, it is very similar to what was observed in (Lambaerts et al. 2012) in the case of baroclinic instability of the upper-layer jet.

The wave activity is measured in the same way as in barotropic and baroclinic dipolar breakdown cases, and is presented in Fig. 25. It is persistent and is much stronger than in the dipolar breakdown case at later stages of the evolution. The wave-field at the peak of wave 


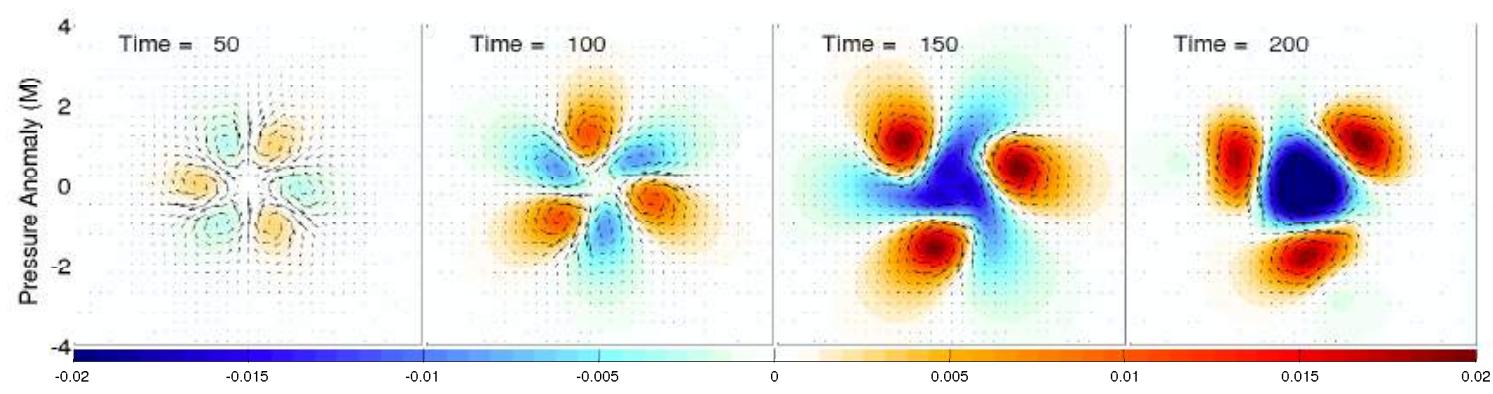

Figure 22. Evolution of pressure anomaly in the "dry" simulation of the development of the baroclinic instability with most unstable mode $l=3$ in the lower layer.
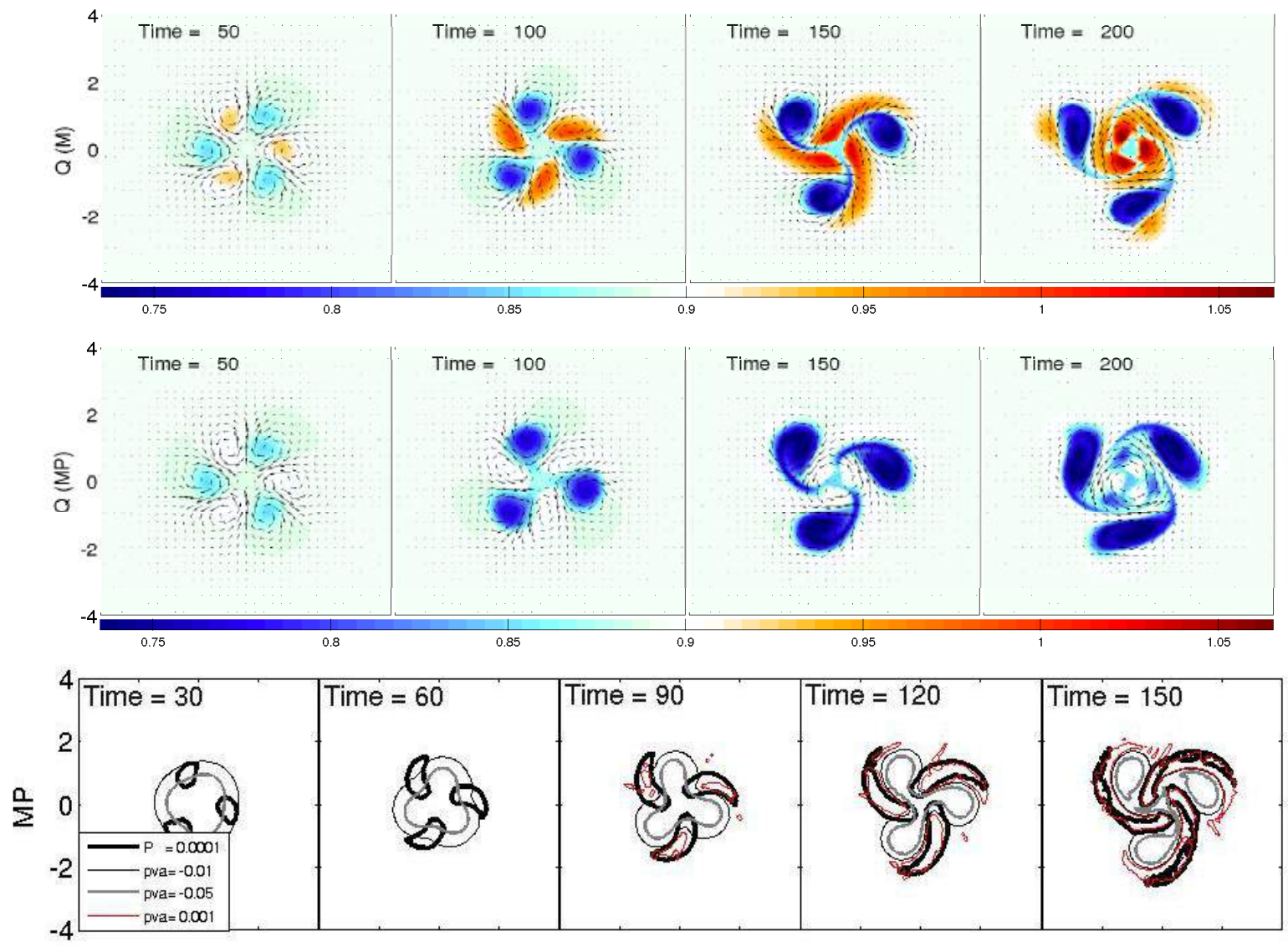

Figure 23. Evolution of moisture in M (top) and MP (middle) environments and joint evolution of PVA and precipitation in the MP simulation (bottom) of a vortex with $l=3$ most unstable mode.

activity of Fig. 25 correspond to a packet of baroclinic IGW propagating out of the vortex (nt shown).

\section{Summary, conclusions, and discussion}

We performed a comparative analysis of development of barotropic and baroclinic instabilities of large-scale small Rossby-number anticyclonic and cyclonic shielded $\alpha$-Gaussian vortices evolving in initially uniform humidity field with moisture being (i) passively advected (M), (ii) 


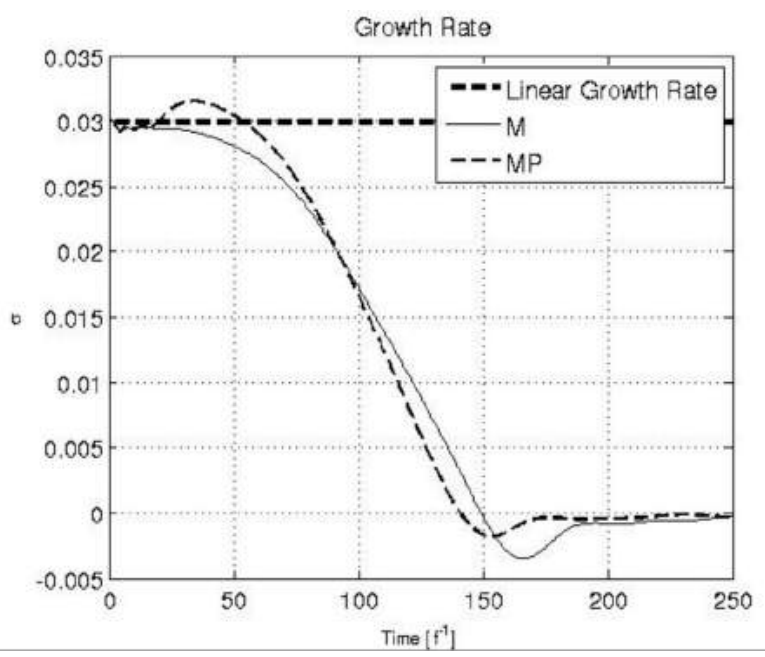

Figure 24. Evolution of the growth rate of the perturbation during the tripolar evolution scenario in M and MP environments.

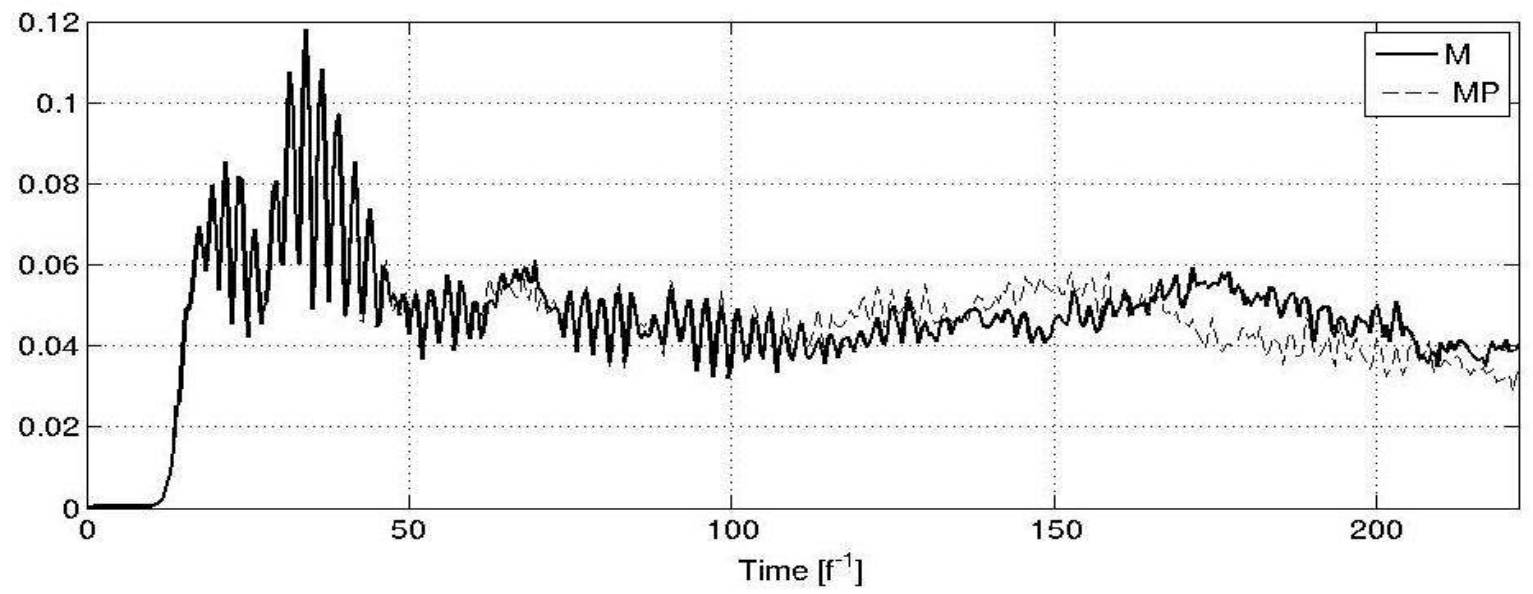

Figure 25. Wave activity during the evolution of the baroclnic instability in tripole formation scenario in $\mathrm{M}$ and $\mathrm{MP}$ environments.

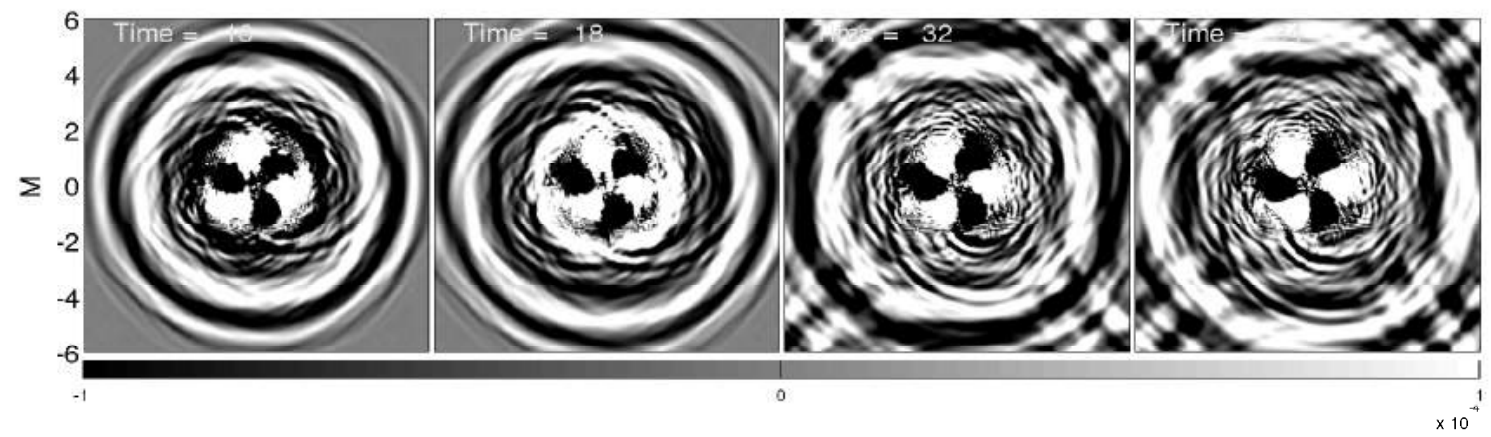

Figure 26. Wave field corresponding to the peak of the wave activity of Fig. 25. 
subject to precipitation beyond a saturation threshold (MP), with corresponding convective flux, (iii) subject to precipitation and evaporation, with two different evaporation parameterisations. At a preliminary stage we accomplished a detailed linear stability analysis of the vortex solutions, which provided unstable modes used for initialisations of direct numerical simulations of the dynamical saturation of the instabilities.

Our general conclusion is that condensation and latent heat release, especially in conjunction with evaporation, considerably modify the evolution of the instabilities. The enhancement of cyclonic component of the flow is universal, although particular modifications, including decrease or increase of the growth rate and of the wave activity, are not universal and are sensitive to the details of the flow configuration. Scenario of evolution of the instability is sensitive to the details of the background vortex flow, in particular its stratification in the two-layer case, with instabilities of different azimuthal structure having close growth rates and evolving and precipitating differently. Strong differences are observed in the evolution of unstable modes with azimuthal numbers $l=2$ and $l=3$, including sensitivity to the effects of moisture. Thus, characteristic "rain bands" were found during the evolution of the tripolar instability of the upper-layer vortex. Therefore, scenario of evolution of the atmospheric vortices, and in particular precipitation patterns, are highly sensitive to the azimuthal structure of the most unstable mode, and the latter is sensitive to fine details of the vortex profile. This is important to bear in mind in the general context of predictability of atmospheric flows. It is worth emphasising in this context the influence of the upper-level dynamics of the atmosphere upon precipitation in the lower layer which we observed. Although it leads to qualitative changes, like e.g. structure and trajectories of secondary dipoles in dipolar splitting scenario, the influence of moist effects upon low Rossby number vortices is less spectacular than for the high Rossby number ones (Lahaye and Zeitlin 2015), where they lead to overall intensification of the vortex and emission of convectively coupled waves. Still, precipitation events enhance the emission of inertia-gravity waves, which is a fact of general importance. Yet, comparing the results of our highly idealised simulations with observations would be a too-far stretch.

To summarize the main results,

- In the barotropic case the linear stability analysis confirmed results of previous studies with similar, albeit different, vortex profiles. Differences in growth rates of unstable modes of the same azimuthal structure for cyclonic and anticyclonic vortices with the same parameters, and thus cyclone-anticyclone asymmetry, were found. The azimuthal structure of the most unstable mode changes when vortex parameters change. Nonlinear simulations of the evolution of the instability showed that a known "dipolar splitting" scenario, which we confirmed in the "dry" (M) environment, is modified by moist effects. Both the structure and the direction of propagation of the resulting dipoles are changed by the moist convection, especially in the presence of evaporation. If evaporation is strong enough, the resulting dipoles can be completely reorganised. A strong cycloneanticyclone asymmetry in the response to the condensation and related convection, with anticyclonic vortex evolution being affected much stronger. At the same time, IGW emission, especially in moist-precipitating and evaporating environment, is substantially stronger for the cyclonic vortex. We also detected an overall destructive influence of precipitation and related convection upon the growth rate of the instability, apart from a short transient increase of the growth rate, which differs from the results of Lambaerts et al. (2011b, 2012). A substantial increase of the IGW activity was detected at the onset of splitting of the initial vortex in two dipoles, in both dry and moist environments, and also at the secondary dipoles' separation stage. The condensation was shown to be at the origin of the latter increase.

- In the baroclinic case the analysis was concentrated on the idealised upper-level cyclonic 
vortices of cut-off low type. Full linear stability analysis revealed sensitivity of the growth rates and of azimuthal structure of the most unstable modes to the vortex parameters, and in particular to the stratification and thickness ratio parameters. Nonlinear simulations with the most unstable mode of the same azimuthal structure $l=2$ as in the barotropic case gave a similar scenario of evolution, with strong enhancement of cyclonic vorticity in the lower humid layer. On the contrary, the gravity wave activity was not enhanced by precipitation events due to the overall low values of velocity in the lower layer. Nonlinear simulations with the most unstable mode with $l=3$ revealed a totally different saturation scenario with secondary tripole formation, specific precipitation patterns ("rain bands") and enhanced inertia-gravity wave activity. Contrary to both barotropic and baroclinic dipolar splitting scenarios of evolution, the growth rate of the perturbation is considerably enhanced by precipitation in this case, exceeding the "dry" linear stability value.

Acknowledgements: We are grateful to N. Lahaye for help with numerical simulations and discussions. We also thank anonymous Referees for careful reading of the manuscript and useful suggestions. 


\section{References}

Baey, J.-M. and Carton, X., Vortex multipoles in two-layer rotating shallow-water flows. J. Fluid Mech., 2002, 460, $151-175$.

Betts, A. K., and Miller, M. J., A new convective adjustment scheme. Part II: Single columns tests using GATE wave, BOMEX, ATEX and arctic air-mass data sets. Quart. J.Roy. Meteor. Soc., 1986, 112,693709.

Bouchut, F., J. , Lambaerts, G. Lapeyre, and Zeitlin, V., Fronts and non-linear waves in a simplified shallow-water model of the atmosphere with moisture and convection. Phys. Fluids, 2009, 21, 116604,doi:10.1063/1.3265970.

Boyd, J. P., Orthogonal rational functions on a semi-infinite interval. J. Comput. Phys., 1987, 70 (1), 63 - 88.

Carton, X. J., Flierl, G. R., and Polvani, L. M., The generation of tripoles from unstable axisymmetric vortex structures. Europhys. Lett., 1989, 9, 339-344.

Gill, A. E., Studies of moisture effects in simple atmospheric models: The stable case. Geophys. Astrophys. Fluid Dyn., 1982, 19, 119 - 152.

Gimeno, L., R. M. Trigo, P. Ribera, and J. A. Garcia., Editorial: Special issue on cut-off low systems (COL). Meteorology and Atmospheric Physics, 2007, 96, 1-2.

Goswami, P., and Goswami, B. N., Modification of $\mathrm{n}=0$ Equatorial Waves Due to Interaction between Convection and Dynamics. J. Atmos. Sci., 1991, 48, 2231 - 2244.

Kloosterziel, R. C., Carnevale, G. F. and Orlandi, P, Inertial instability in rotating and stratified fluids: barotropic vortices. J. Fluid Mech., 2007, 583, 379 - 412.

Kondo, J., Saigusa, N. and Sato, T., A parameterization of evaporation from bare soil surfaces. J. Appl. Met., 291990, , pp 385-389.

Lahaye, N. and Zeitlin, V., Centrifugal, barotropic and baroclinic instabilities of isolated ageostrophic anticyclones in the two-layer rotating shallow water model and their nonlinear saturation. J. Fluid Mech., 2015, 762 , pp 5-34

Lahaye, N. and Zeitlin, V., Understanding instabilities of tropical cyclones with moist-convective rotating shallow-water model, 2016, J. Atmos. Sci.,73, 497-523 .

Lambaerts, J., Lapeyre, G., Zeitlin, V. and Bouchut, F., Simplified two-layer models of precipitating atmosphere and their properties. Phys. Fluids, 2011, 23, 046603.

Lambaerts, J., Lapeyre, G., and Zeitlin, V., Moist versus Dry Barotropic Instability in a Shallow-Water Model of the Atmosphere with Moist Convection. J. Atmos. Sci., 2011, 68, 1234-1252.

Lambaerts, J., Lapeyre, G., and Zeitlin, V., Moist versus dry baroclinic instability in a simplified two-layer atmospheric model with condensation and latent heat release, J. Atmos. Sci., 2012, 69, 1405-1426.

Lapeyre, G., and Held, I. M., The role of moisture in the dynamics and energetics of turbulent baroclinic eddies. J. Atmos. Sci., 2004, 61, 1693 - 1710.

Neelin, J. D., Held, I. M., and K. H. Cook, Evaporation-wind feedback and low frequency variability in the tropical atmosphere. J. Atmos. Sci., 1987, 44, 2341-2348.

Ooyama, K., Numerical simulation of the life-cycle of tropical cyclone. J. Atmos. Sci., 1969, 26, 3-40.

Schecter, D.A, and Dunkerton, T. J., Hurricane formation in diabatic Ekman turbulence. Quart. J. Roy. Meteor. Soc., 2009, 135, 823.

Trefethen, L. N., Spectral Methods in MATLAB. SIAM, 2000.

Zeitlin, V., Decoupling of balanced and unbalanced motions and inertia-gravity wave emission: Small versus large Rossby numbers. J. Atmos. Sci.,2008, 65, 3528 - 3542.

Zehnder, J.A., A comparison of convergence- and surface-flux-based convective parameterizations with applications to tropical cyclogenesis. J. Atmos. Sci., 2001, 58, No. 3, 283-301 